\title{
Realistic simulations of gravitational lensing by galaxy clusters: extracting arc parameters from mock DUNE images
}

\author{
M. Meneghetti ${ }^{1,2}$, P. Melchior ${ }^{2}$, A. Grazian ${ }^{3}$, G. De Lucia ${ }^{4}$, K. Dolag ${ }^{4}$, M. Bartelmann ${ }^{2}$, \\ C. Heymans ${ }^{5,9}$, L. Moscardini ${ }^{6,7}$, and M. Radovich ${ }^{8}$ \\ 1 INAF - Osservatorio Astronomico di Bologna, via Ranzani 1, 40127 Bologna, Italy \\ e-mail: massimo.meneghetti@oabo.inaf.it \\ 2 ITA, Zentrum für Astronomie, Universität Heidelberg, Albert Überle Str. 2, 69120 Heidelberg, Germany \\ 3 INAF - Osservatorio Astronomico di Roma, via di Frascati 33, 00040 Roma, Italy \\ ${ }^{4}$ Max-Planck-Institut für Astrophysik, Karl-Schwarzschild-Str. 1 85748, Garching bei Muenchen, Germany \\ 5 Department of Physics and Astronomy, The University of British Columbia, 6224 Agricultural Road, Vancouver, V6T 1Z1, Canada \\ ${ }^{6}$ Dipartimento di Astronomia, Università di Bologna, via Ranzani 1, 40127 Bologna, Italy \\ 7 INFN-National Institute for Nuclear Physics, Sezione di Bologna, viale Berti Pichat 6/2. 40127 Bologna, Italy \\ 8 INAF - Osservatorio Astronomico di Capodimonte, Salita Moiarello 16, 80131 Napoli, Italy \\ 9 Institut d'Astrophysique de Paris, UMR 7095 CNRS, 98bis Bd Arago, 75014 Paris, France
}

Received 21 November 2007 / Accepted 10 January 2008

\section{ABSTRACT}

\begin{abstract}
Aims. We present a newly developed code that allows simulations of optical observations of galaxy fields with a variety of instruments. The code incorporates gravitational lensing effects and is targeted at simulating lensing by galaxy clusters. Our goal is to create the tools required for comparing theoretical expectations with observations to better understand of how observational noise affects lensing applications such as mass estimates, studies of the internal properties of galaxy clusters and arc statistics.

Methods. Starting from a set of input parameters, characterizing both the instruments and the observational conditions, the simulator provides a virtual observation of a patch of the sky. It includes several sources of noise such as photon-noise, sky background, seeing, and instrumental noise. Ray tracing through simulated mass distributions accounts for gravitational lensing. Source morphologies are realistically simulated based on shapelet decompositions of galaxy images retrieved from the GOODS-ACS archive. According to their morphological class, spectral-energy-distributions are assigned to the source galaxies to reproduce observations of each galaxy in arbitrary photometric bands.

Results. We illustrate our techniques showing virtual observations of a galaxy-cluster core as it would be observed with the space telescope DUNE, which was recently proposed to ESA within its "Cosmic vision" program. We analyze the simulated images using methods applicable to real observations and measure the properties of gravitational arcs. In particular, we focus on the determination of their length, width, and curvature radius.

Conclusions. We find that arc properties strongly depend on several properties of the sources. In particular, our results show that compact, faint, or low surface-brightness galaxies that are barely detectable are more easily distorted as arcs with large length-towidth ratios. We conclude that realistic lensing simulations can be obtained with the method proposed here. They will be essential for evaluating and improving the analysis techniques currently used for cosmological interpretations of cluster lensing.
\end{abstract}

Key words. gravitational lensing - galaxies: clusters: general - cosmology: observations - methods: numerical - submillimeter

\section{Introduction}

Gravitational lensing of distant galaxies is one of the most powerful tools for investigating the content of the universe and for understanding how the matter is distributed within the cosmic structures. As the most massive objects in the universe, galaxy clusters are the most powerful existing gravitational lenses, which makes them suitable for a large number of purposes. Clusters are able to magnify distant galaxies, making them natural gravitational telescopes, that can be used to investigate the first sources of light in the universe (Ellis et al. 2001; Schaerer \& Pello 2003; Kneib et al. 2004; Li et al. 2007).

Strong lensing features, like gravitational arcs and multiple images, are observed in the central, densest regions of the lenses (Fort et al. 1988; Lynds \& Petrosian 1989), so they can be used to constrain the inner structure of clusters (e.g. Li et al. 2007; Comerford et al. 2006; Oguri et al. 2003; Sand et al. 2004; Meneghetti et al. 2007, 2005a).
Gravitational arcs are also used in a variety of cosmological applications. Their frequency on the sky reflects the abundance, the concentration, and the redshift distribution of massive lenses in the universe. Moreover, it has been shown that the probability of forming arcs is enhanced during cluster mergers (Torri et al. 2004; Fedeli et al. 2006). Thus, arc statistics are another powerful tool for tracing structure formation and for constraining the cosmological parameters (Bartelmann et al. 1998; Li et al. 2005; Meneghetti et al. 2005b). Moreover, since they form along critical lines, whose size scales with the source and lens redshifts, lensed images arising from multiple sources at different redshifts can be used to constrain the geometry of the universe (Golse et al. 2002; Meneghetti et al. 2005a), and thus also cosmological parameters.

In the outer regions of clusters, the distortions induced by the cluster potentials are weaker. Nevertheless, using suitable algorithms (e.g. Bartelmann \& Schneider 2001, and references 
therein), the distortion field can be inverted to obtain twodimensional surface-density maps for the lenses (see Clowe et al. 2004; Starck et al. 2006; Paulin-Henriksson et al. 2007, for some recent examples). Strong and weak lensing can also be used jointly to improve the mass reconstrution in a non-parametric way (Bradac et al. 2004; Cacciato et al. 2006; Diego et al. 2007).

Although the potential power of gravitational lensing for the above-mentioned applications has been already demonstrated, different implementations of methods adopted for extracting the desired information from the lensing data often give contradictory results. An example is given by the so-called "arc statistics problem", i.e. the claimed inconsistency between the observed and the predicted number of highly distorted gravitational arcs expected to be seen on the sky in the standard $\Lambda$ CDM cosmology (Bartelmann et al. 1998; Dalal et al. 2005; Wambsganss et al. 2004; Luppino et al. 1999; Gladders et al. 2003; Zaritsky \& Gonzalez 2003; Li et al. 2005). Moreover, testing a particular analysis technique and/or comparing the results to the theoretical expectations is difficult because observational noise introduces uncertainties that need to be properly modeled (see e.g. Rhodes et al. 2007).

Following an approach that turned out to be extremely successful in cosmic shear studies (see e.g. Heymans et al. 2006; Massey et al. 2007a), we aim at overcoming these difficulties and facilitating the comparison between theory and observations by performing realistic simulations that include the relevant observational effects. Using suitable ray-tracing codes, the gravitational lensing produced by realistic mass distributions obtained from numerical $N$-body and hydrodynamical simulations can be included. Simulated images can finally be analyzed like real observations.

In this paper, we describe a simulation pipeline that we use to simulate observations of lensing events in galaxy clusters, but it can also be easily used for simulating lensing by large-scale structures in wide fields. These simulations will be used in forthcoming papers to evaluate the accuracy of the several lensing techniques mentioned above.

The plan of the paper is as follows. In Sect. 2 we present the architecture of the simulator and describe the methods used to include lensing effects and observational noise. In Sect. 3, we use a galaxy cluster obtained from $N$-body simulations and use our code to create mock optical observations of its central regions. We discuss how several strong lensing features can be extracted from the simulated images and how the properties of gravitational arcs can be measured. We also quantify how these properties depend on the assumed source models. Finally, in Sect. 4 we report our conclusions.

\section{The simulator}

In this section we describe the features included in our simulator for optical observations. In the following we assume that:

- the lenses that are eventually placed in front of the sources are obtained from $N$-body and hydrodynamical simulations. However, any deflector can be used for testing the codes, for example, mass distributions that can be generated using analytic models;

- a method for calculating the deflection angle fields out of the simulated mass distributions has been separately implemented.

Our simulator is coupled to an existing ray-tracing code that has been widely used in the past (see e.g. Meneghetti et al. 2000, 2001, 2003, 2005c) and that will be described in Sect. 3.2.
We also implemented other routines for calculating the deflection angles from a distribution of particles using a tree algorithm (similar to that described in Aubert et al. 2007) and fastFourier-transform methods (Bartelmann \& Meneghetti 2004). Ray-tracing through multiple planes for mimicking the effects of the large-scale-structure of the universe has also been implemented (Pace et al. 2007). The choice of the implementation is not important for this paper, but it will become more relevant in future applications of this code. In general, for a ray-tracing simulation through a single plane of matter, given a deflection angle map $\hat{\boldsymbol{\alpha}}$, the angular position $\boldsymbol{y}$ where a light ray is emitted can be easily calculated from the apparent position $\boldsymbol{x}$ through the lens equation,

$\boldsymbol{y}=\boldsymbol{x}-\frac{D_{\mathrm{ls}}}{D_{\mathrm{s}}} \hat{\boldsymbol{\alpha}}(\boldsymbol{x})$

where all angles are referred to an arbitrarily chosen optical axis passing through the observer. The angular diameter distances between the lens and the source, $D_{\mathrm{ls}}$, and between the observer and the source, $D_{\mathrm{s}}$, have been used to re-scale the deflection angles to the proper lens-source configuration.

Assuming we know the distribution of matter along the line of sight and that we are able to characterize its deflection field, we proceed now to generate a population of source galaxies, to assign them a given morphology and surface brightness distribution, to distort them according to the lensing effect produced by the matter between the observer and the sources, and finally to visualize the results of a virtual observation including all relevant sources of noise and the convolution with a particular instrument.

\subsection{Galaxy positions, luminosities, and redshifts}

The virtual observation comprises a user-specified field-of-view. This defines the size of the light-cone along which source galaxies are distributed. When generating galaxy positions, we randomly choose the projected position of the sources on the sky. Their orientation is also randomly selected. Thus, we do not consider, at the moment, clustering of galaxies and intrinsic alignments that could represent a potential source of errors in weak lensing measurements, but we will address this issue in future work.

The source distances, morphological types, and intrinsic magnitudes are generated using observed luminosity functions per given redshift bin. We chose to adopt the luminosity functions derived from the VIMOS VLT Deep Survey (VVDS, Le Fèvre et al. 2005), for which different versions exist for four principal spectral types of galaxies up to $z=1.5$.

Zucca et al. (2006) have divided the galaxies into four types, corresponding to E/S0 (type 1), early spiral (type 2), late spiral (type 3) and irregular template (type 4). This work was done by matching the UBVRI magnitudes with empirical sets of spectralenergy-distributions (SEDs) (Arnouts et al. 1999). These SEDs are shown in Fig. 1 in different colors and were used to characterize the flux of the synthetic galaxies in our simulations.

The luminosity function for each spectral type and redshift bin is of the form

$$
\begin{aligned}
\phi(M) \mathrm{d} M= & 0.4 \phi^{\star} \ln 10\left(10^{0.4\left(M^{\star}-M\right)}\right)^{\alpha+1} \\
& \cdot \exp \left(-10^{0.4\left(M^{\star}-M\right)}\right) \mathrm{d} M
\end{aligned}
$$

It gives the number of galaxies per unit magnitude and per cubic Mpc (Schechter 1976). The characteristic absolute magnitude 


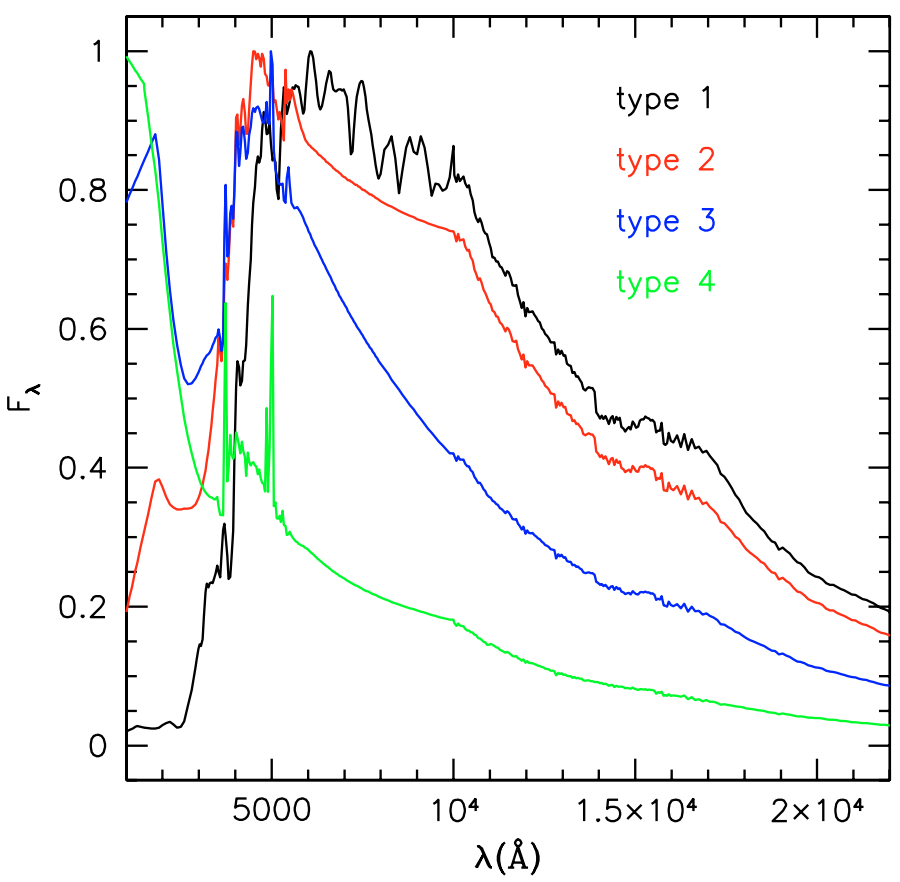

Fig. 1. Spectral-energy distributions used to characterize the galaxies of different spectral types. The galaxies are divided into four principal classes: ellipticals/S0 (type 1), early spirals (type 2), late spirals (type 3 ), and irregulars (type 4). The wavelengths are given in $\AA$, while flux is arbitrary.

$M^{\star}$, the characteristic density $\phi^{\star}$, and the slope $\alpha$ are determined by fitting the observed magnitude distributions of galaxies with the same spectral type and with spectroscopic redshift in the interval between $z_{\min }$ and $z_{\max }$. We used the values reported in Table 3 of Zucca et al. (2006) for the rest frame $B$-band (Johnson $B$ filter). The galaxies used in the simulations were generated so as to reproduce the observed luminosity functions in this reference band. The magnitudes in the other bands were easily calculated by using the previous SEDs, suitably normalized such as to assign the correct luminosity in the $B$-band, and by convolving them with the appropriate filter curves.

The luminosity functions for different morphological types exist for galaxies up to $z=1.5$. For higher redshifts, we adopted the luminosity functions published by Ilbert et al. (2005) and by Paltani et al. (2007) and we extrapolated the fractions of ellipticals, spirals, and irregulars from those observed at lower redshift. In particular at $z \gtrsim 2$, we assumed that only $5 \%$ of the galaxies are ellipticals or S0, while the vast majority of them are spirals and irregulars.

Certainly, important assumptions are made in the process of generating the population of galaxies filling the light cones. First, the VVDS is limited to $I$-magnitude 24 ( $A B$ magnitude). Thus, the faint tail of the distribution of galaxy luminosities is extrapolated from a fit performed on brighter galaxies. Second, the absolute magnitudes in bands different from the reference $B$-band are calculated by adopting a limited number of SEDs. Third, as explained earlier, we can use luminosity functions per different spectral types only at $z<1.5$. For larger redshifts, we must use a single luminosity function and extrapolate the fractions of galaxies with a given SED from lower redshift.

Although it is natural to expect a dominant fraction of irregulars and spirals at high redshift, our procedure may lead to significant errors, especially when simulating deep observations. To check and validate our methods, we made the following test. We generated a population of galaxies as described above and compared the number counts per magnitude bin and squared arcmin to those measured in the deep observations like the HUDF. We find good agreement between the number counts independent of the band selected for the experiment. For example, we show in the left panel of Fig. 2 the comparison between our prediction based on the VVDS luminosity functions and the HUDF in the $z$-band (F850LP filter on board the Hubble telescope). Although the selected band is much redder than the reference band and the size of the HUDF is rather small, the curves are almost perfectly superposed, confirming the reliability of our results despite the assumptions.

\subsection{Galaxy surface-brightness distributions}

The light emission from galaxies falling into the telescope field of view is modeled using shapelet functions. This approach has been used by Massey et al. (2004), although with a different shapelet decomposition method. According to Refregier (2003), the galaxy two-dimensional surface-brightness distribution, $I(\boldsymbol{x})$, can be approximated by a summation over a set of basic functions,

$I(\boldsymbol{x})=\sum_{n_{1}, n_{2}=0}^{\infty} I_{\boldsymbol{n}} B_{\boldsymbol{n}}\left(\boldsymbol{x}-\boldsymbol{x}_{\mathrm{c}} ; \beta\right)$,

where $\boldsymbol{x}_{\mathrm{c}}$ indicates the galaxy centroid, $\boldsymbol{x}=\left(x_{1}, x_{2}\right)$ is a twodimensional vector, and $\boldsymbol{n}=\left(n_{1}, n_{2}\right)$. The basic functions $B_{n}(\boldsymbol{x} ; \beta)$ are called shapelets and have the form

$B_{n}(\boldsymbol{x}, \beta)=\beta^{-1} \phi_{n_{1}}\left(\beta^{-1} x_{1}\right) \phi_{n_{2}}\left(\beta^{-1} x_{2}\right)$,

where the one-dimensional functions $\phi_{n}(x)$ are related to the Gauss-Hermite polynomials, $H_{n}(x)$,

$\phi_{n}(x)=\left[2^{n} \pi^{1 / 2} n !\right]^{-1 / 2} H_{n}(x) \exp \left(-x^{2} / 2\right)$.

The parameter $\beta$ defines the scale of the galaxy image. The coefficients $I_{n}$ properly weight the contribution of each shapelet function to the surface brightness of the galaxy and are given by

$I_{n}=\int \mathrm{d}^{2} x I(\boldsymbol{x}) B_{n}(\boldsymbol{x} ; \beta)$

Basic photometric information about the galaxy is readily derived from the shapelet coefficients. For example, the total flux $I$ is given by

$I=\pi^{1 / 2} \beta \sum_{n_{1}, n_{2}}^{\text {even }} 2^{\frac{1}{2}\left(2-n_{1}-n_{2}\right)}\left(\begin{array}{c}n_{1} \\ n_{1} / 2\end{array}\right)^{1 / 2}\left(\begin{array}{c}n_{2} \\ n_{2} / 2\end{array}\right)^{1 / 2} I_{\boldsymbol{n}}$,

and the rms radius $r_{I}$, defined as $r_{I}^{2} \equiv \int \mathrm{d}^{2} x x^{2} I(\boldsymbol{x}) / I$, is given by

$$
\begin{aligned}
r_{I}^{2}= & \pi^{1 / 2} \beta^{3} I^{-1} \sum_{n_{1}, n_{2}}^{\text {even }} 2^{\frac{1}{2}\left(4-n_{1}-n_{2}\right)}\left(1+n_{1}+n_{2}\right) \\
& \times\left(\begin{array}{c}
n_{1} \\
n_{1} / 2
\end{array}\right)^{1 / 2}\left(\begin{array}{c}
n_{2} \\
n_{2} / 2
\end{array}\right)^{1 / 2} I_{n}
\end{aligned}
$$

The shapelet decomposition has proven to be a powerful technique for image anaysis (see e.g. Melchior et al. 2007; Massey et al. 2007b; Goldberg \& Bacon 2005). Among the many applications, Kelly \& McKay (2004) have shown that it can be applied to morphological classification. In fact, they find that galaxies of different morphological types separate well in 

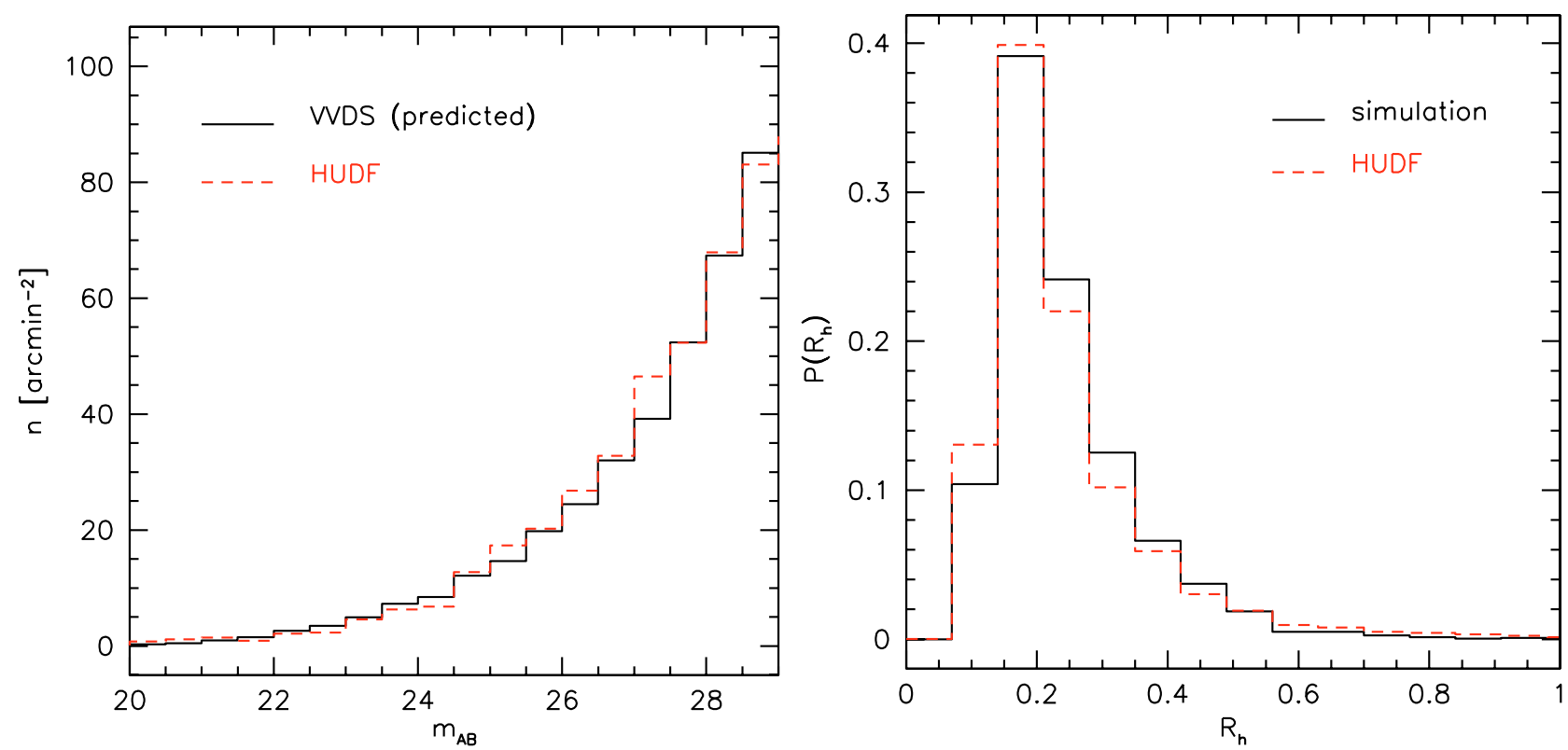

Fig. 2. Left panel: number counts per apparent magnitude bin and squared arcmin predicted by our method (solid line) and observed in the HUDF (dotted line). Magnitudes are given in the $z$-band. Right panel: probability distributions of the galaxy half-light radii in the HUDF (dashed histogram) and in a field simulated with Skylens (solid histogram).

shapelet space. On the other hand, Massey et al. (2004) show that an infinite number of synthetic galaxy images can be easily created from a set of shapelet coefficients obtained from decomposing real galaxies. Following these ideas, we aim at using real shapelet decompositions to characterize galaxy morphologies in our simulator. In detail, we proceed as follows:

1. we use the newly developed code by Melchior et al. (2007) for shapelet-decomposing the images of 3000 galaxies from the GOODS HST/ACS data (Giavalisco et al. 2004). For each galaxy, the code provides the best fit set of coefficients, $I_{n}^{\mathrm{GOODS}}$, together with their errors and the scale parameter, $\beta^{\mathrm{GOODS}}$;

2. for about half of the galaxies in the database, a spectral classification is available, which allows us to distinguish between morphological types and to assign an SED to each galaxy. For those galaxies with no classification, we assume that they are irregulars or late spirals, since they are typically faint and distant blue objects. Based on this classification, the galaxy decompositions are divided into four catalogs corresponding to the SEDs described in Sect. 2.1;

3. aiming at generating a galaxy of type $\mathrm{M}$, one entry from the corresponding catalog is randomly chosen. Since our catalogs contain a limited number of galaxies, which may be smaller than the number of galaxies to be generated in the simulation, replications of the same galaxy may occur. To avoid that, whenever a galaxy is chosen, we slightly change the values of the shapelet coefficients (within their errors) to obtain a new, slightly different, galaxy image. This procedure must be employed with caution. Although there is no correlation between the coefficients in a single galaxy decomposition by construction, as the basis functions are orthogonal, it must be considered that galaxies of differing morphological types separate cleanly in shapelet space (see e.g. Kelly \& McKay 2004). Thus, we might end up with unusual objects if the coefficients were changed such that the new galaxies are too distant from the original ones in shapelet space. In particular, this may happen when the starting galaxy decompositions are affected by large uncertainties. Such a problem may bias the size distribution of our simulated galaxies. To check whether the differences between the simulated and the observed distributions of galaxy sizes shown in the right panel of Fig. 2 are due to our method to avoid image replications, we ran a simulation without modifying the coefficients of the decompositions. We did not find any improvement in the comparison with the observed galaxy sizes, meaning that the differences are caused merely by number effects. On the other hand, we used a sample of high$S / N$ galaxies in these experiments, whose decompositions are quite robust;

4. the galaxy image is finally rescaled on the basis of the input flux. From the decompositions contained in our shapelet database, we build an empirical relation between the scale parameter and the flux. Then, we use this relation and its dispersion to draw the scale parameter $\beta$ that corresponds to the input flux $I$. Equation (7), however, states that $I \propto \beta I_{n}$. Thus, to ensure consistency, when scaling a GOODS galaxy with scale parameter $\beta_{0}$ and flux $I_{0}$ to one with scale parameter $\beta$ and observed flux $I$, we also adjust its shapelet coefficients by multiplying them by $\left(I / I_{0}\right)\left(\beta_{0} / \beta\right)$. To verify the reliability of our method, we compared the size distribution of simulated galaxies with that observed in the HUDF. The probability distribution functions of the half-light radii (as outputted by SExtractor, Bertin \& Arnouts 1996) in the simulated and in the observed HUDF are shown in the right panel of Fig. 2. For the observed HUDF, we refer to the catalog published by Beckwith et al. (2006). The simulated images were analyzed consistently, i.e. by adapting the SExtractor parameters to those used in the observations. Clearly, the distributions are very similar, although the half-light radii tend to be slightly larger in the simulation (median $R_{\mathrm{h}}=0.211^{\prime \prime}$ ) than in the real observation (median $R_{\mathrm{h}}=0.204^{\prime \prime}$ ). An even more accurate result will be achieved when our shapelet database is extended using deeper observations, including a larger number of low-flux sources;

5. once a set of coefficients and a scale parameter have been defined, they can be used to generate a new galaxy 

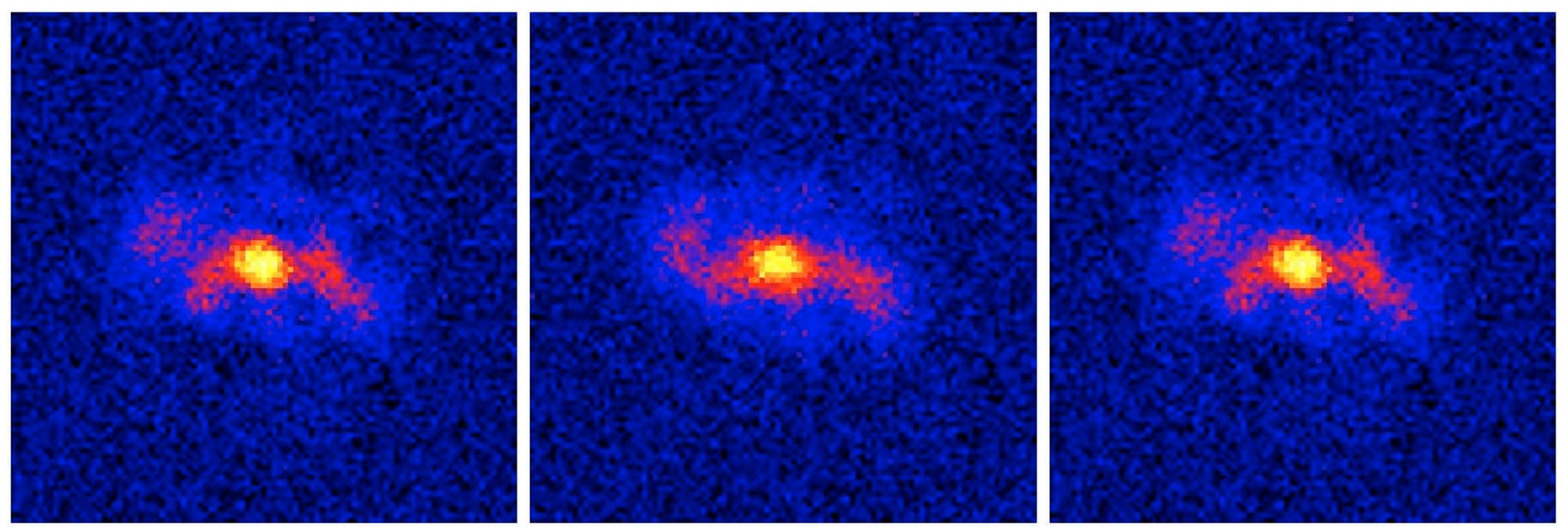

Fig. 3. Example of generation of synthetic galaxies with shapelets. In the left panel the original image of a GOODS galaxy is shown. The galaxy is decomposed into $9 \times 9$ shapelets and reconstructed in the central panel. A second galaxy is then generated from the same shapelet decomposition by slightly modifying the coefficients and displayed in the right panel. The same color scale is used for all three images. To reproduce the background and the noise properties of the image in the left panel, we simulated an observation with HST/ACS (filter F850LP, $t_{\mathrm{exp}}=10600 \mathrm{~s}$ ). Each frame is $2.9^{\prime \prime}$ on a side.

surface-brightness distribution. In order to ensure random orientations, we rotate by a random angle $\psi \in[0, \pi]$.

In Fig. 3 we show an example illustrating the generation of syntetic galaxies out of a real GOODS galaxy. In the left panel, we show a spiral galaxy from GOODS. The galaxy is decomposed into shapelets with maximum order $n_{1}=n_{2}=9$. The decomposition is then used to reconstruct the original galaxy image in the central panel. Finally, a second galaxy surface brightness distribution is generated from the same decomposition but after having slightly changed the values of the shapelet coefficients. As explained above, the coefficients and the scale parameter may also be rescaled to change the output flux of the sources, allowing us to create synthetic galaxies in arbitrary numbers. Moreover, as will be shown later, using shapelets permits us to easily incorporate lensing effects in the simulations. All images already contain several sources of noise that will be discussed in the following sections.

\subsection{Observing deep fields}

Once a number of galaxies have been generated, having a luminosity, an SED, a redshift, and a shape assigned, we can simulate observations for a particular instrumental set-up. This implies calculating the number of photons coming from a patch of the sky that are collected by each element of the CCD camera, after the light has passed through a sequence of mirrors and filters, and, eventually, through the Earth's atmosphere.

\subsubsection{Photon counts}

For the implementation of our simulator, we followed the prescriptions given by Grazian et al. (2004) in the construction of the Large-Binocular-Telescope Camera Image Simulator (LBCSIM). Given a telescope of diameter $D$, the number of photons collected by the CCD pixel at $\boldsymbol{x}$ in the exposure time $t_{\text {exp }}$, from a source whose surface brightness is $I(\boldsymbol{x})$ $\left(\mathrm{erg} \mathrm{s}^{-1} \mathrm{~cm}^{-2} \mathrm{~Hz}^{-1} \operatorname{arcsec}^{-2}\right.$ ), is

$n_{\gamma}(\boldsymbol{x})=\frac{\pi D^{2} t_{\exp } p^{2}}{4 h} I(\boldsymbol{x}) \int \frac{T(\lambda)}{\lambda} \mathrm{d} \lambda$, where $p$ is the pixel size in arcsec, $h$ is the Planck constant, and $T(\lambda)$ the total transmission, which is given by the product of the atmospheric extinction for a given airmass, $A^{\prime}(\lambda)=$ $10^{-0.4 \text { airmass } \cdot A(\lambda)}$ (airmass $=0$ for observations from space), and the efficiencies of the CCD, $C(\lambda)$, of the filter, $F(\lambda)$, of the mirror, $M(\lambda)$, and of the optics $O(\lambda)$,

$T(\lambda)=A^{\prime}(\lambda) \cdot C(\lambda) \cdot F(\lambda) \cdot M(\lambda) \cdot O(\lambda) \cdot$

The number of photons is converted into a number of ADUs (astronomical data units) by dividing by the gain $g$ of the CCD:

$$
A D U(\boldsymbol{x})=\frac{n_{\gamma}(\boldsymbol{x})}{g} .
$$

\subsubsection{Lensing effects}

Lensing by intervening matter along the line of sight is applied by mapping the CCD pixel coordinates using the lens Eq. (1). The pixel at $\boldsymbol{x}$ is now connected to the position $\boldsymbol{y}$ on the plane where the galaxy surface brightness is assigned. Then, the surface brightess at $\boldsymbol{y}$ is converted into ADUs using Eqs. (9) and (11), and the computed value is assigned to the pixel at position $\boldsymbol{x}$ on the CCD:

$A D U_{\text {lensed }}(\boldsymbol{x})=A D U[\boldsymbol{y}(\boldsymbol{x})]$.

Note that in the strong lensing regime one coordinate $\boldsymbol{y}$ can correspond to more than one pixel coordinate $\boldsymbol{x}$.

The application of Eq. (12) in the case of arbitrary pixel scales involves two steps. First, since the deflection angles may be determined on a grid whose point positions generally differ from those of CCD pixels, the deflection angle map must be interpolated at each pixel position, $\boldsymbol{x}$. We use a bi-cubic interpolation scheme, which works well provided the spatial resolution of the virtual CCD is not too high compared to that of the input deflection angle map. Second, since the galaxies are distributed in redshift, the deflection angles must be rescaled by the factor $D_{\mathrm{ls}} / D_{\mathrm{s}}$ appearing in Eq. (1) for each source. Especially when simulating observations of large fields, this operation may become time-consuming because of the large number of pixels and sources. To shorten the calculations, we bin the sources in redshift and project all the galaxies in a given redshift bin on a single 
plane. For example, we assign the redshift $z_{\mathrm{s}}=\left(z_{\min }+z_{\max }\right) / 2$ to all galaxies at a redshift between $z_{\min }$ and $z_{\max }$. By changing the size of the redshift bins, one can increase or decrease the number of source planes. All sources falling into the same redshift bin can then be processed at the same time, saving a considerable amount of computational time.

Note that using the shapelet formalism for representing the source galaxies allows weak lensing transformations to be applied through relatively simple operators acting on the shapelet states. For first-order lensing, Refregier (2003) shows that the transformation can be written as

$A D U_{\text {lensed }}(\boldsymbol{x}) \simeq\left[1+\kappa(\boldsymbol{x}) \hat{K}+\gamma_{i}(\boldsymbol{x}) \hat{S}_{i}\right] A D U(\boldsymbol{x})$,

where $\kappa$ and $\gamma=\left(\gamma_{1}, \gamma_{2}\right)$ are the convergence and the shear, respectively. The operators $\hat{K}$ and $\hat{S}$ can be expressed in terms of the raising and lowering operators for the quantum harmonic oscillator as

$$
\begin{aligned}
\hat{K} & \equiv 1+\frac{1}{2}\left[\hat{a}_{1}^{\dagger 2}+\hat{a}_{2}^{\dagger 2}-\hat{a}_{1}^{2}-\hat{a}_{2}^{2}\right] \\
\hat{S}_{1} & \equiv \frac{1}{2}\left[\hat{a}_{1}^{\dagger 2}-\hat{a}_{2}^{\dagger 2}-\hat{a}_{1}^{2}+\hat{a}_{2}^{2}\right] \\
\hat{S}_{2} & \equiv \hat{a}_{1}^{\dagger 2} \hat{a}_{2}^{\dagger 2}-\hat{a}_{1}^{2} \hat{a}_{2}^{2} .
\end{aligned}
$$

Thus, by using these formulas in combination with Eqs. (3), (9), and (11), the lensing distortion can be expressed in terms of transformations of the shapelet coefficients, $I_{n}$.

\subsubsection{Convolution with the PSF and seeing}

Several sources of noise can then be added to the image to resemble real observational conditions. First, the images can be convolved with the instrumental point-spread-function (PSF):

$I^{\prime}(\boldsymbol{x})=\int \mathrm{d}^{2} x^{\prime} I\left(\boldsymbol{x}^{\prime}\right) F\left(\boldsymbol{x}-\boldsymbol{x}^{\prime}\right)$.

The convolution can be done in two ways. In analogy to the galaxy images, a given PSF model, $F(\boldsymbol{x})$, can be decomposed into shapelets. Convolution is entirely analytically possible in shapelet space, given the invariance of the shapelet functions under Fourier transformations. The full formalism is explained in Refregier (2003). This approach has been used by Massey et al. (2007a) for image simulations. The method is efficient for doing convolutions involving un-lensed or weakly lensed galaxies, i.e. galaxies with known shapelet decompositions. For very distorted galaxies, like in the strong lensing regime, applying this method would require further shapelet-decomposing the lensed images, whose shapelet coefficients cannot be easily derived from the unlensed ones (for example using Eq. (13)). This would then lead to a loss of computational efficiency. For this reason, when simulating fields including strong lensing features, we opt for applying standard FFT techniques for doing convolution operations. This approach allows the shape of the PSF to be simulated very realistically. However, the PSF is unique for the whole image; i.e. local variations in the PSF shape have been not mimicked so far.

In weak lensing applications, the PSF needs to be corrected to extract the lensing signal from the source ellipticities. Residuals in the PSF corrections, due for example to the abovementioned variations in the PSF shape, are one of the major sources of error (see e.g. Rhodes et al. 2007).

To take this into account in our simulations, we propose the following approach to modeling the PSF. The general idea is that distortions of the PSF shape can be described as an additional "lensing" effect. Anisotropies cause the images of stars to acquire irregular shapes, being stretched along some particular directions as if an external shear was applied. Such an external shear can be characterized by a power spectrum, whose amplitude defines the intensity of the anisotropies and whose shape characterizes the spatial scales over which it varies.

Let the shear power spectrum be $P_{\gamma}$. We can link the power spectrum to a source's potential power spectrum using standard definitions. Since the components of the shear are combinations of the second derivatives of the lensing potential,

$$
\begin{aligned}
& \gamma_{1}=\frac{1}{2}\left(\frac{\partial^{2} \psi}{\partial x_{1}^{2}}-\frac{\partial^{2} \psi}{\partial x_{2}^{2}}\right) \\
& \gamma_{2}=\frac{\partial^{2} \psi}{\partial x_{1} \partial x_{2}},
\end{aligned}
$$

in Fourier space, we find that

$$
\begin{aligned}
& \hat{\gamma}_{1}=-\frac{k_{1}^{2}-k_{2}^{2}}{2} \hat{\psi} \\
& \hat{\gamma}_{2}=-k_{1} k_{2} \hat{\psi},
\end{aligned}
$$

where the hat denotes Fourier transforms and $\boldsymbol{k}=\left(k_{1}, k_{2}\right)$ is the wave vector. The power spectrum of the source potential is thus

$P_{\psi}=\frac{4}{k^{4}} P_{\gamma}$

Using this formalism, the distortions of the PSF can be introduced by applying additional lensing to the images already convolved with a PSF function. Given a potential $\psi$, the corresponding deflection angle field is

$\alpha=\nabla \psi$,

and in Fourier space

$\alpha_{1}=-\mathrm{i} k_{1} \hat{\psi}$

$\alpha_{2}=-\mathrm{i} k_{2} \hat{\psi}$.

Thus, the power spectra of the two components of the deflection angle are

$P_{\alpha_{1}}=k_{1}^{2} P_{\psi}=\frac{k_{1}^{2}}{k^{4}} P_{\gamma}$
$P_{\alpha_{2}}=k_{2}^{2} P_{\psi}=\frac{k_{2}^{2}}{k^{4}} P_{\gamma}$.

For simplicity, we assume that $P_{\gamma}$ is a Gaussian,

$P_{\gamma}(k)=A_{\gamma} \exp -\frac{k^{2}}{k_{\text {cut }}^{2}}$,

whose amplitude is $A_{\gamma}$ and whose characteristic scale is $k_{\text {cut }}$. This last parameter sets the scales over which spatial variations of the PSF shape occur.

Thus, we use $P_{\gamma}$ to generate maps of the deflection angle components. For doing that we assume that these maps are Gaussian, with zero mean and variance defined by the amplitude of the input power spectrum. The deflection angles are used to lens the images as explained in the previous section. When simulating ground-based observations, seeing is simulated by further convolving the image with a Gaussian $G$ of size $\sigma$ :

$I_{\sigma}(\boldsymbol{x})=\int \mathrm{d}^{2} x^{\prime} I^{\prime}\left(\boldsymbol{x}^{\prime}\right) G\left(\boldsymbol{x}-\boldsymbol{x}^{\prime}\right)$ 


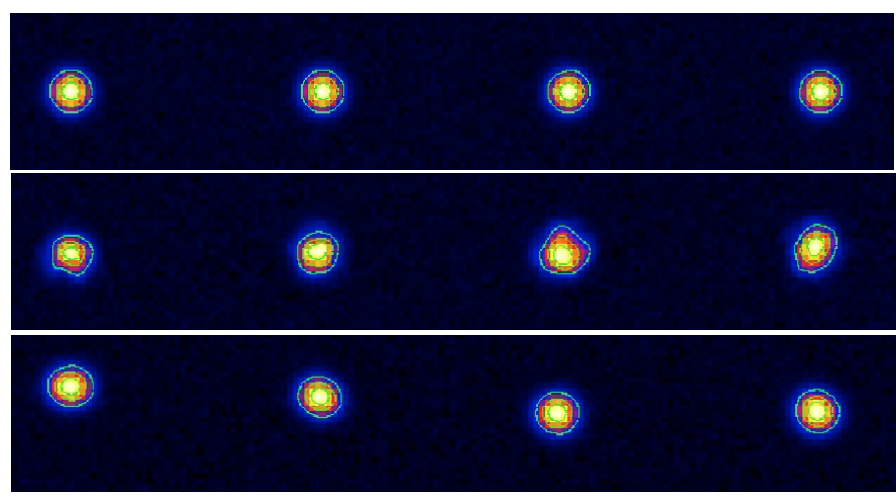

Fig. 4. Images of an array of stars observed with an LBT-like telescope. The angular separation between the stars is $10^{\prime \prime}$ along the $x$ axis. In the top panel, an isotropic PSF $\left(F W H M=0.6^{\prime \prime}\right)$ is mimicked. In the middle panel, anisotropies varying on scales of $\sim 1^{\prime \prime}$ are included. In the bottom panel we simulate large-scale variations of the PSF shape on scales of $\sim 15^{\prime \prime}$.

In Fig. 4 we show some examples. In the top panel, an array of stars convolved with an isotropic PSF with Gaussian profile and FWHM of $0.6^{\prime \prime}$ (including seeing) is shown. The angular separation of the stars along the $x$ axis is $10^{\prime \prime}$. In the middle panel, we introduce variations of the PSF shape on scales of $\sim 1^{\prime \prime}$. The amplitude of the distortions is intentionally quite large and corresponds to $A_{\gamma}=0.9$. Instead, in the bottom panel we mimic a PSF that slowly varies on scales of $\sim 15^{\prime \prime}$. These examples show that by properly choosing the parameters $A_{\gamma}$ and $k_{\text {cut }}$, a suitable level of noise in the PSF can be added.

\subsubsection{Sky background and photon noise}

The number of ADUs per pixel from the sky background are given by

$A D U_{\text {sky }}=\frac{\pi D^{2} t_{\exp } p^{2}}{4 h g} \int \frac{T(\lambda) S(\lambda)}{\lambda} \mathrm{d} \lambda$,

where $S(\lambda)$ is the sky flux per square arcsec at a given wavelength and $T(\lambda)$ is calculated now at airmass $=0$. The sky flux is modeled differently for observations from the ground and from space. For ground-based observations, we use different night sky spectra according to the different phases of the moon. The spectra are those used in Grazian et al. (2004). They correspond to $0,3,7,10$, and 14 days after the dark moon. For simulating the sky as seen from the space, we adopt several spectra of zodiacal light, representing the typical variations of the zodiacal background radiation and of the earthshine (Giavalisco et al. 2002).

Photon noise is assumed to be Poissonian. For each pixel the noise (in ADUs) is generated by adding random deviates with Gaussian probability distribution of mean zero and width

$$
\begin{aligned}
\sigma_{N}(\boldsymbol{x})= & \left\{n_{\exp }\left(\frac{R O N}{g}\right)^{2}+\frac{A D U(\boldsymbol{x})+A D U_{\text {sky }}}{g}\right. \\
& \left.+\left(f+\frac{a^{2}}{n_{\exp }^{2}}\right)\left[A D U(\boldsymbol{x})+A D U_{\text {sky }}\right]^{2}\right\}^{1 / 2} .
\end{aligned}
$$

In the previous formula $R O N$ is the read-out noise of the chip, $n_{\exp }$ is the number of exposures, and $a$ the flat-field term, which we fix at $a=0.005$ following Grazian et al. (2004). The term $f$ indicates the flat-field accuracy, which is determined by the
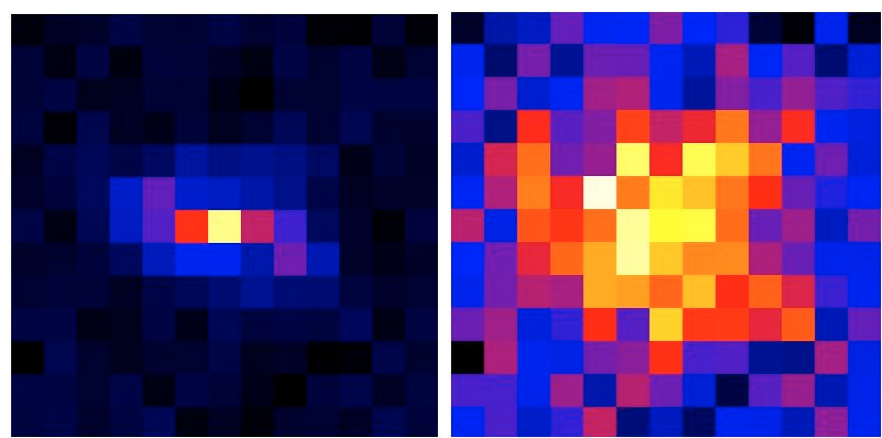

Fig. 5. The same galaxy displayed in the central panel of Fig. 3 is shown here as it should be observed in the $I$-band by LBT with a diffractionlimited PSF (left panel) and in bad seeing conditions ( $\sigma=1.2^{\prime \prime}$, right panel). The exposure time is $t_{\exp }=2000 \mathrm{~s}$ and the airmass is 1 . The scale of each frame is $2.9^{\prime \prime}$.

number of flat-field exposures and by the level of the sky background as

$f=\left(N_{f f} \cdot B \cdot g\right)^{-1}$.

A more detailed explanation of these formulas is given in Sect. 4.2 of Grazian et al. (2004).

\subsubsection{Producing the images}

By properly setting the total efficiency (using the correct efficiency curves for optics, mirrors and CCDs) observations from several instruments can be simulated. As an example, we show in Fig. 5 how the same galaxy displayed in the central panel of Fig. 3 would appear when observed with the LBT with a diffraction limit PSF (left panel) and in bad seeing conditions ( $\sigma=1.2^{\prime \prime}$; right panel). The exposure time is $2000 \mathrm{~s}$ with airmass $=1$. The pixel size of the LBT camera is $0.224^{\prime \prime}$, in contrast to the $0.03^{\prime \prime}$ of HST/ACS (after drizzling). The spiral structure of the galaxy is thus not resolved by LBT. Seeing blurs the image, destroying most of the remaining morphological information.

Combining several galaxies into the same field, we were able to produce artificial galaxy fields. One example is given in Fig. 6, where we simulated a deep field of $1^{\prime} \times 1^{\prime}$ observed with the HST/ACS with an exposure time of $10600 \mathrm{~s}$. In this simulation, no lensing effects were included. As demonstrated here, by using our procedure for generating artificial galaxies we can produce very realistic simulated images of the sky.

\subsubsection{Foregrounds}

The light emission from foreground galaxies can be included in simulated images adopting the same procedure employed for background sources. We note that cluster galaxies in particular represent a potential problem for lensing analyses. For weak lensing studies, misidentification of cluster members as background sources can bias the shear measurements. In addition the bright galaxies concentrated near the cluster center (which corresponds to the strong lensing regime) complicate the detection of lensed sources.

In order to include realistic galaxy clusters in our simulated images, we used results from a semi-analytic model of galaxy formation coupled to a cluster $N$-body re-simulation. The semianalytic model used in this study was described in De Lucia \& Blaizot (2007), and we refer to the original study (and references 


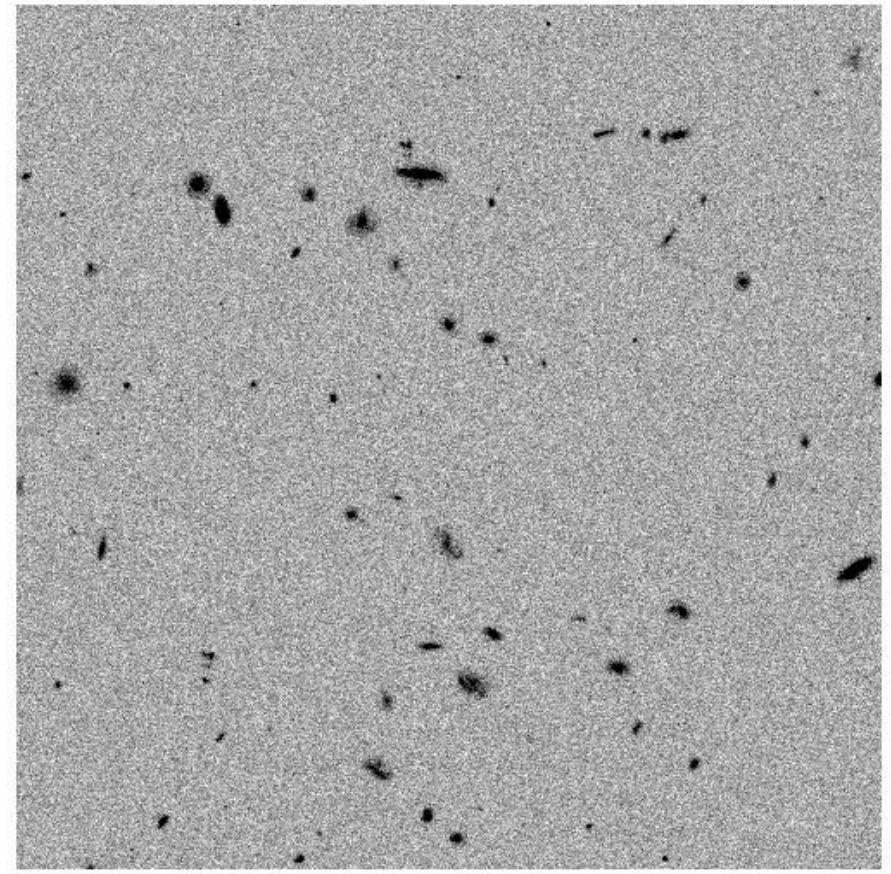

Fig. 6. Simulation of a deep field observed by HST/ACS in the $z$-band. The exposure time is $t_{\exp }=10600 \mathrm{~s}$ and the field of view is $1^{\prime} \times 1^{\prime}$.

therein) for more details about the physical processes modeled. The model is used to generate a catalog containing positions and luminosities of model galaxies at different redshifts. This information is used to simulate galaxy images as described in the previous section. Again, we determine the morphology of our model galaxies by using the $B$-band, bulge-to-disc ratio, together with the observational relation by Simien \& de Vaucouleurs (1986) between this quantity and the galaxy morphological type.

An example of a simulated observation of a galaxy cluster is shown in Fig. 7. The cluster is at redshift $z \sim 0.3$. It is observed with HST/ACS in the $z$-band with an exposure time of $1000 \mathrm{~s}$. The cluster is dominated by some very bright galaxies $\left(L \sim 10^{12} L_{\odot}\right)$ in the central part. They are expected to significantly complicate the observability of lensing features, in particular radial arcs, occurring near the cluster center.

\section{Applications of the method}

In this section, we discuss one possible application of our simulator, i.e. simulating gravitational arcs in the center of galaxy clusters. Then we apply some techniques that can also be used in real observations to measure the properties of the arcs detected in the images.

\subsection{The cluster model}

The cluster used in this paper is one the clusters previously studied by Dolag et al. (2005). Its lensing properties were also investigated by Puchwein et al. (2005) and by Meneghetti et al. (2007). A detailed description of the simulation can be found in these papers. Its reference name is $g 1$. Although several versions of this cluster exist that include the treatment of the gas component with several physical processes, we chose to use here the simplest simulation, where the cluster contains only dark matter particles.

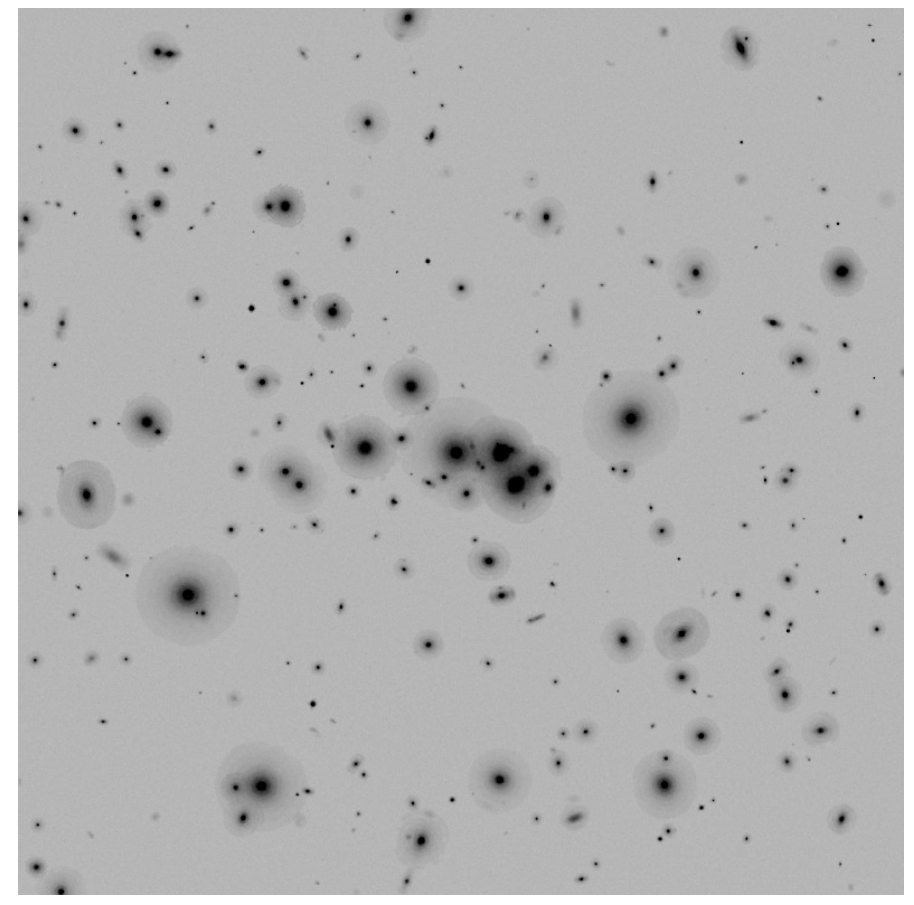

Fig. 7. Virtual observation in the $z$-band by HST/ACS of a galaxy cluster at $z \sim 0.3$. The exposure time is $t_{\exp }=1000 \mathrm{~s}$ and the field of view is $100^{\prime \prime} \times 100^{\prime \prime}$. The spatial distribution and luminosities of the cluster galaxies have been obtained from semi-analytic models. The lensing effects are disabled in this simulation.

The halo has a mass of $\sim 1.4 \times 10^{15} h^{-1} M_{\odot}$ (resolved by more than a million particles within the virial region), thus it represents a very massive and efficient strong lens at $z=0.3$. We chose this redshift because it is close to where the strong lensing efficiency of clusters is the highest for sources at $z_{\mathrm{s}} \gtrsim 1$ (Li et al. 2005).

Although we are investigating the lensing property of this halo only at $z=0.3$, during the simulation, 92 time slices were saved from redshift 60 to 0 . These are equidistant in time. For each snapshot, we computed group catalogs and their embedded substructures using a standard friends-of-friends algorithm with a linking length of 0.2 in units of the mean particle separation and a modified version of the algorithm SUBFIND (Springel et al. 2001) that was extended for future applications to hydro-simulations. Substructure catalogs were then used to construct merger history trees as described in Springel (2005) and De Lucia \& Blaizot (2007). These merger trees are the basic input needed for the semi-analytic model described in Sect. 2.3.6. A total of 402 sub-halos were found within a cube of $3 h^{-1} \mathrm{Mpc}$ comoving side length. The mass enclosed in these sub-halos amounts to $\sim 7 \times 10^{13} h^{-1} M_{\odot}$. Using the predictions of the semianalytic model, the virial region of the cluster was populated with 1645 galaxies.

The surface density map corresponding to one projection of the cluster is shown in Fig. 8. The size of the image is $\left(1.5 h^{-1} \mathrm{Mpc}\right)^{2}$. The inner region appears quite elliptical and devoid of large substructures. Secondary massive clumps of matter are located at distances $\gtrsim 400 h^{-1} \mathrm{kpc}$ from the cluster center.

\subsection{Calculations of deflection angles}

Ray-tracing simulations were carried out using the technique described in detail in several earlier papers (e.g. Bartelmann et al. 1998; Meneghetti et al. 2000). We selected a cube of $3 h^{-1} \mathrm{Mpc}$ 


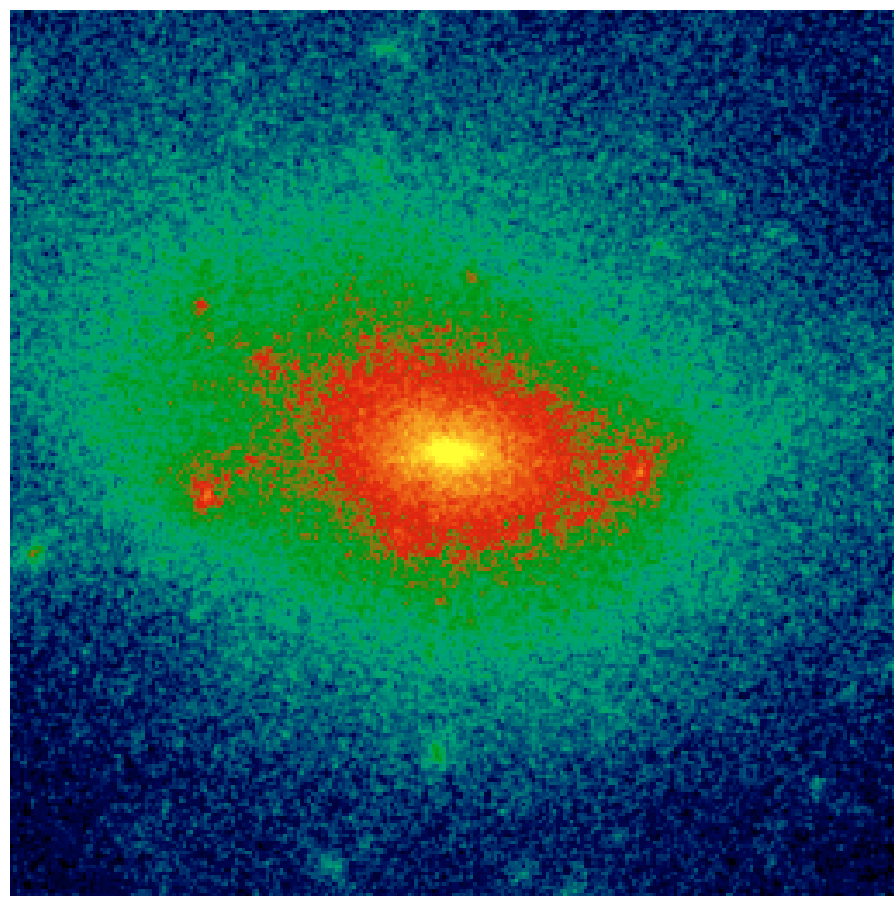

Fig. 8. Surface density map of cluster $g 1$ corresponding to projecting the cluster along the $z$-axis of the simulation box. The side-length of the image is $1.5 h^{-1} \mathrm{Mpc}$ comoving (corresponding to $\sim 372^{\prime \prime}$ ).

comoving side length, centered on the halo center and containing the high-density region of the cluster. The particles in this cube were used for producing a three-dimensional density field, by interpolating their position on a grid of $1024^{3}$ cells using the Triangular Shaped Cloud method (Hockney \& Eastwood 1988). Then, we projected the three-dimensional density field along the coordinate axes, obtaining three surface-density maps $\Sigma_{i, j}$, used as lens planes in the following lensing simulations.

The lensing simulations were performed by tracing a bundle of $2048 \times 2048$ light rays through a regular grid, covering the central sixteenth of the lens plane. This choice was driven by the necessity of studying the central region of the cluster in detail, where critical curves form, taking the contribution into account from the surrounding mass distribution to the deflection angle of each ray.

Deflection angles on the ray grid were computed as follows. We first define a grid of $256 \times 256$ "test" rays, for each of which the deflection angle was calculated by directly summing the contributions from all cells on the surface density map $\Sigma_{i, j}$,

$\boldsymbol{\alpha}_{h, k}=\frac{4 G}{c^{2}} \sum_{i, j} \Sigma_{i, j} A \frac{\boldsymbol{x}_{h, k}-\boldsymbol{x}_{i, j}}{\left|\boldsymbol{x}_{h, k}-\boldsymbol{x}_{i, j}\right|^{2}}$,

where $A$ is the area of one pixel on the surface density map and $\boldsymbol{x}_{h, k}$ and $\boldsymbol{x}_{i, j}$ are the positions on the lens plane of the "test" ray $(h, k)$ and of the surface density element $(i, j)$. We avoided the divergence when the distance between a light ray and the density grid point was zero by shifting the "test" ray grid by half-cells in both directions with respect to the grid on which the surface density is given. We then determined the deflection angle of each of the $2048 \times 2048$ light rays by bi-cubic interpolation between the four nearest test rays.

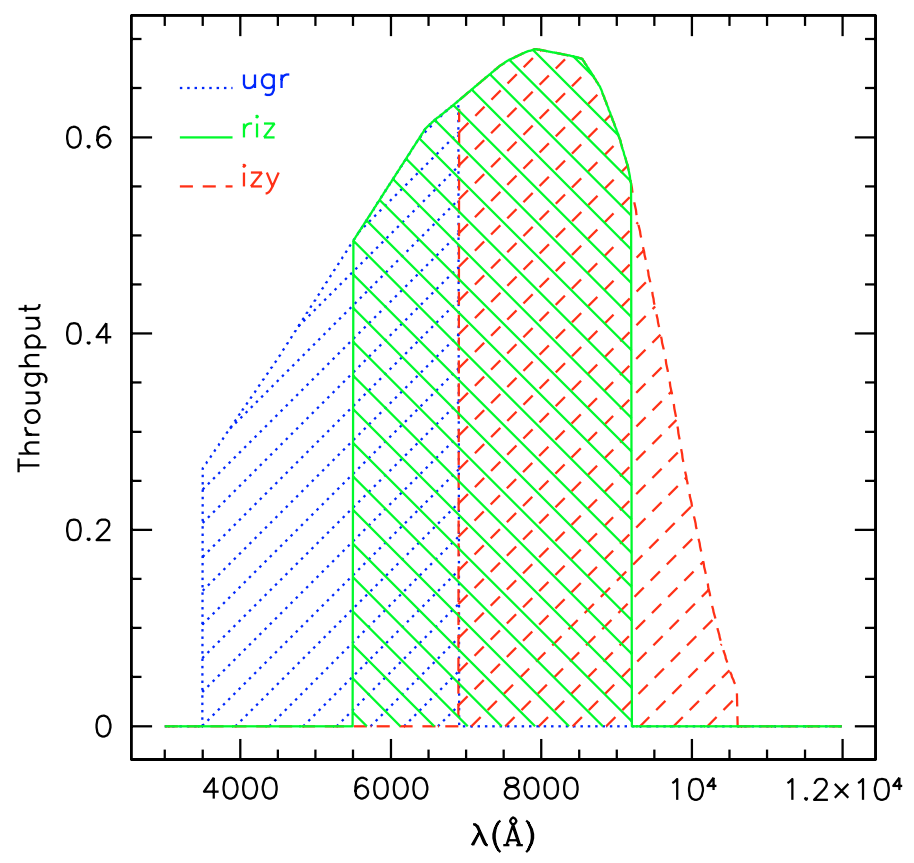

Fig. 9. Throughputs assumed in carrying out the simulations with the DUNE satellite. Three broad-band filters are used: ugr (dashed lines), riz (solid lines), izy (courtesy of Amara, Rhodes and the DUNE collaboration members). Note that the figure does not reflect the final choice for the filters to be mounted on DUNE (see text for more details).

\subsection{Observations}

For simulating observations of our numerical cluster, we considered an instrument with the characteristics of the planned space telescope DUNE (Dark Universe Explorer; http://www . dune-mission.net). This mission has been recently proposed to ESA within its "Cosmic Visions" program. It is targeted at the dark components of the universe with a wide-field imager, in particular through weak lensing. However, thanks to the good spatial resolution, the panoramic field of view ( 0.5 square degrees), and high sensitivity, this instrument will be extremely useful for many other studies including strong lensing, galaxy formation and evolution, baryonic acoustic oscillations, and even planet searches.

The motivation for adopting a future instrument for our virtual observation is twofold. On one hand, we would like to illustrate that the simulator is a powerful tool for testing techniques that are commonly applied to observational data. On the other hand, we would like to highlight that codes like this can help in defining the scientific tasks that can be fulfilled by future missions.

DUNE is planned to have a mirror diameter of $1.2 \mathrm{~m}$, a spatial resolution of $0.10^{\prime \prime} / \mathrm{pixel}$ in the visible, and a total efficiency close to $\sim 70 \%$. During the proposal preparation, three broadband filters were used, namely a $u+g+r(u g r)$, an $r+i+z$ (riz) and an $i+z+y(i z y)$ filter. In the proposed version, DUNE will not provide 3 bands in the visible. On the contrary, there will be one filter in the visible (riz) and 3 filters in the NIR $(Y, J, H)$. The tests that are discussed below were performed using the riz filter. Just for the purpose of illustrating the capabilities of the simulator for mimicking observations in multiple bands, we used the $u g r$ and the izy filters (see Fig. 10 below). The throughputs that we assume, including the quantum efficiency of the detector and the optical design, are shown in Fig. 9. The PSF is expected to have an FWHM of $0.23^{\prime \prime}$ with ellipticity lower than $6 \%$. 


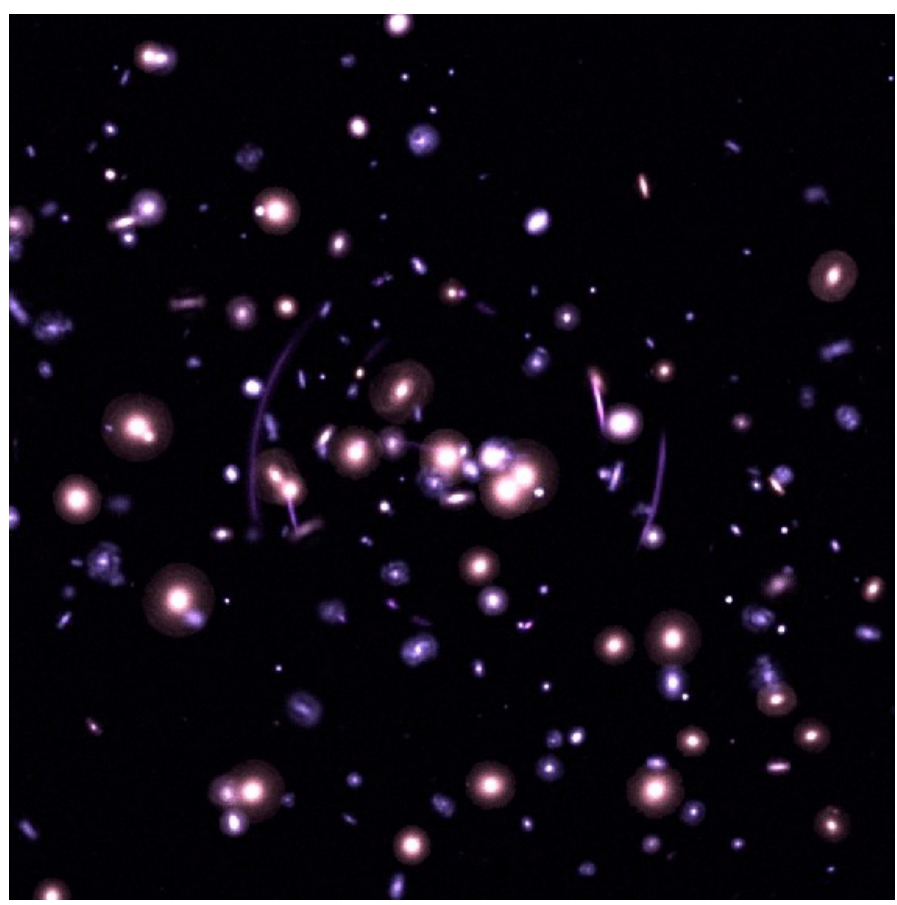

Fig. 10. Composite $u g r+r i z+i z y$ image of the cluster $g 1$. Several strong lensing features are present in the image. The FOV size is $100^{\prime \prime} \times 100^{\prime \prime}$.

A virtual observation of the inner $100^{\prime \prime} \times 100^{\prime \prime}$ region of cluster $g 1$ is shown in Fig. 10. It is a composite ugr, riz and izy image, corresponding to an exposure time of $1000 \mathrm{~s}$. In these tests, we assume an exposure time of $1000 \mathrm{~s}$, although it has been proposed that DUNE exposures will be longer (1500 s). The galaxies were drawn from our the shapelet library, as explained earlier. To facilitate the formation of strong lensing features, we intentionally placed seven "test" galaxies close to the caustics of the lens. They are shown in the left panel of Fig. 11, where we run a simulation without the foreground lens and zoom over the region where the caustics are located. The caustics are also shown in blue. For simplicity, we assumed that the "test" sources are all at $z_{s}=1.5$. Two galaxies, labeled $\mathrm{A}$ and $\mathrm{B}$, are placed very close to the cusps of the tangential caustic, so they are expected to produce extended cusp arcs. Two other galaxies $(F, G)$ are placed along the radial caustic and their images will be radially elongated arcs. The remaining "test" sources $(\mathrm{C}, \mathrm{D}, \mathrm{E})$ are located along the fold of the tangential caustic, so they should be lensed to form fold arcs. The lensed images are presented in the central panel of Fig. 11.

Some of the arcs, and especially the radial ones, are partially or totally hidden by the light of the foreground galaxies, as can be seen in Fig. 10. On the basis of the cluster merger tree, the semi-analytic models predict the presence of very bright galaxies near the cluster center. These render accurate measurements of arcs shapes behind them problematic. In real cases, these galaxies should be removed (see e.g. Sand et al. 2004). Thus, we subtracted the foreground galaxies from the image by fitting their surface brightness profiles with Sersic profiles. This can be done with suitable software like GALFIT (Peng et al. 2002).

We show the image after removing the foreground light in the central panel of Fig. 11. Superposed on the lensed field, we display the cluster critical lines. Cluster members were identified by selecting galaxies with magnitude $m_{r i z}<22$ and rms radius $r_{I}>0.5^{\prime \prime}$. Comparing to the input catalogs of cluster members, we find that foreground galaxies were identified efficiently by applying these selection criteria. The images of the "test" galaxies displayed in the left panel are indicated by letters followed by numbers corresponding to their multiplicity. Note the radial image labeled as G1 (one of the images of source G) is not detectable even after subtracting of the foreground galaxies.

\subsection{Arc properties}

In some applications of strong cluster lensing, such as arc statistics, it is important to accurately measure the properties of gravitational arcs. Properties like length, width and curvature radius of the images can be used to constrain cosmological parameters (Bartelmann et al. 1998, 2003; Wambsganss et al. 2004; Oguri et al. 2003; Li et al. 2005; Meneghetti et al. 2005c; Horesh et al. 2005; Hilbert et al. 2007) but also to determine the innerstructure of galaxy clusters (Sand et al. 2005). In this section we illustrate a procedure for measuring the shape of arcs that can be applied to both real and simulated data, thus favoring the comparison between observations and simulations.

First, we identified the pixels in the image that belong to the lensed images. To do that, we made local measurements of the sky background and set a limit above which pixels can safely be assigned to the arcs. For avoiding the inclusion of noise, especially where foreground galaxies had been subtracted, we selected only pixels above $S / N>5$. The result of this selection is presented in the right panel of Fig. 11. Several images cannot be classified as arcs (for example the images C2, D2, etc.). Thus we excluded them from the following analysis. We focused on the $\operatorname{arcs} \mathrm{A} 1, \mathrm{~B} 1, \mathrm{C} 1, \mathrm{D} 1, \mathrm{E} 1$, and F1.

As suggested by Miralda-Escude (1993; see also Bartelmann et al. 1998; Meneghetti et al. 2000, 2001), we defined three characteristic points on the arcs, namely

1. the brightest point, that is likely to be an image of the source center;

2. the point at the largest distance from 1);

3. the point at the largest distance from 2).

In the case of arc A1, these three points are shown in Fig. 12. We traced a circle segment through these characteristic points. Its length and curvature radius are identified to the length and the curvature radius of the arc, respectively.

Measuring the arc width is not as simple. Arcs are typically structured, as they originate from mergers of multiple images of the same source. Thus, even in the case of simple, elliptical sources, the width is not constant along the arc. Moreover, arcs form in crowded regions of clusters. As shown in the previous examples, the light from cluster members can influence the detectability of the lensed images or of part of them, thus affecting the measurements of the arc widths and lengths. Cluster galaxies can also alter the shape of arcs by means of their lensing effects (Meneghetti et al. 2000). Last but not least important, photon noise causes the edges of the images to be irregular.

The transversal width profile of arc A1 is shown in Fig. 13. This has been recovered by making radial scans of the arc along straight lines passing through the center of curvature CC (see Fig. 12) and intercepting the circular segment CP2-CP3 at equispaced angular separations. The angle is given in degrees with respect to the line CC-CP2. At each scan, we measured the maximal distance between arc points intercepted by the straight line. Some large-scale fluctuations of the width can be recognized, which are caused by mergers of multiple images. To make these large-scale modes more visible, we interpolate the profile with a Bezier curve (Knuth 1986). The profile has several intervals with 

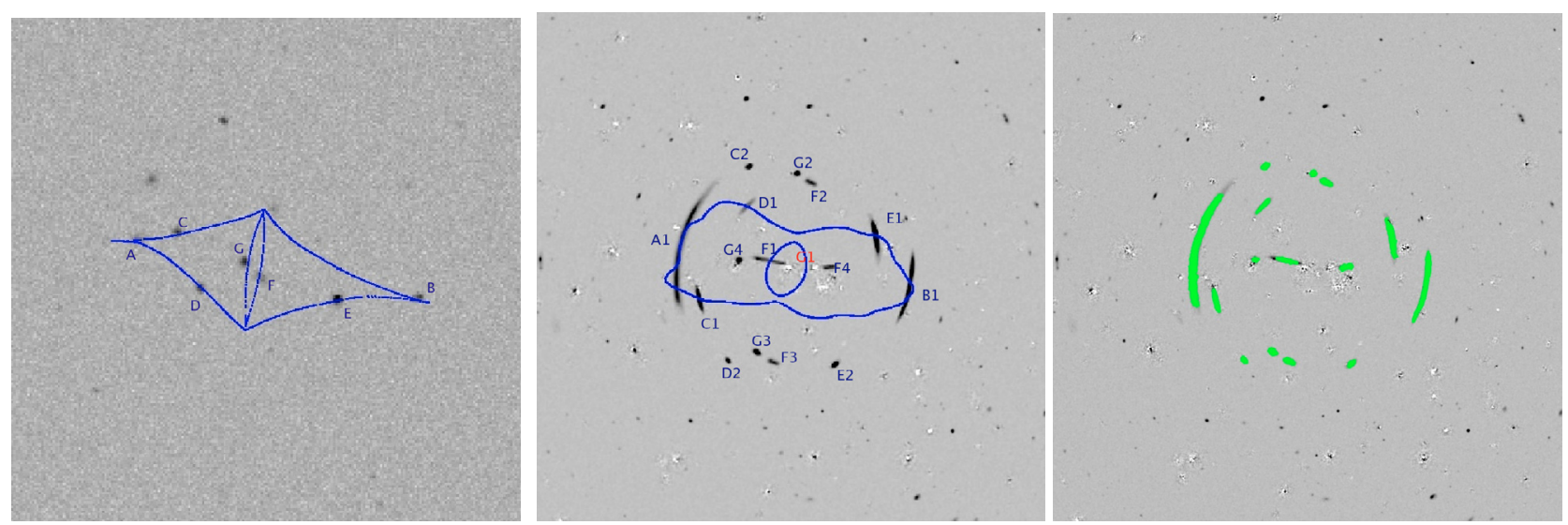

Fig. 11. Left panel: positions of the sources relative to the caustics of the lens. The image is $25^{\prime \prime} \times 25^{\prime \prime}$ wide. Central panel: riz image of the cluster g1 after subtracting the cluster galaxies using magnitude and size cuts (see text for more details). The size of the image is $100^{\prime \prime} \times 100^{\prime \prime}$. The images of the sources in the left panel are indicated by the letters, followed by numbers corresponding to image multiplicity. The image G1 is not visible, but we mark the position where it should be in red. Right panel: the points belonging to the lensed images are marked in green.

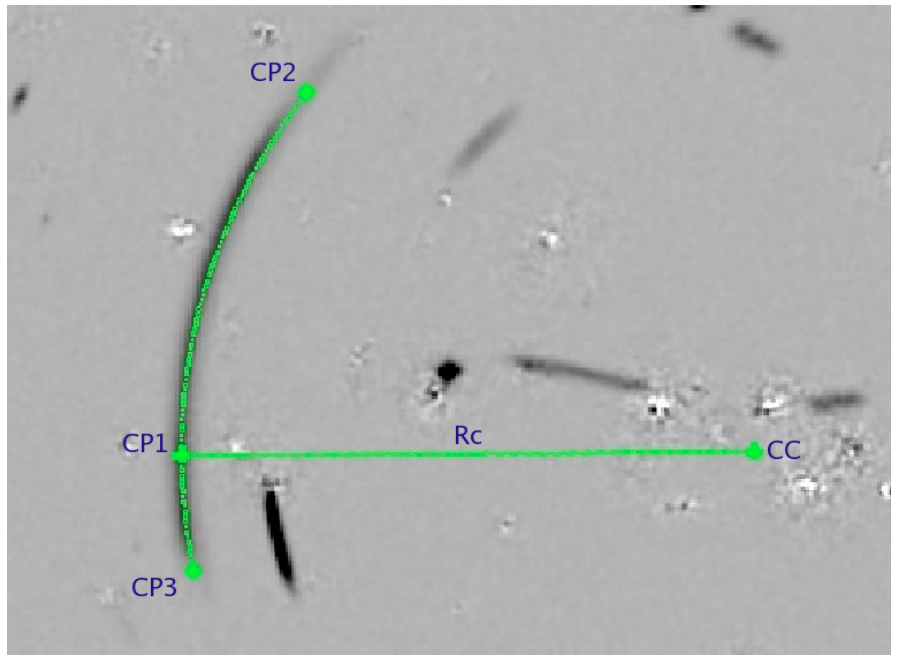

Fig. 12. Fit of the arc A1: three characteristic points have been identified on the image (CP1-3), through which a circular segment (CP2-CP3) has been traced. The curvature radius $(\mathrm{Rc})$ and the center of curvature $(\mathrm{CC})$ of the circular segment define the radius and the center of the arc.

positive curvature separated by valleys. The valleys are located at the positions where the arc crosses the cluster's critical line, as shown in the central panel of Fig. 11. They correspond roughly to the regions where multiple images of the source have merged. Additionally, many other small-scale fluctuations are produced by noise. Thus, it appears difficult to define at which position the arc width has to be measured. We propose here to use the median width.

In the case of observations through the Earth atmosphere, this effect is enhanced as both the width and the length of arcs are affected by the instrumental PSF and by the seeing. Since by definition arcs have large length-to-width ratios, the blurring of the images produces a much larger relative change in the widths than of in lengths. Thus, a correction must be applied to the width measurements in particular. This correction can be made by means of a de-convolution. Let assume that the radial profile of the arc is well fitted by a Gaussian,

$G(x)=Q \exp -\frac{(x-\bar{x})^{2}}{2 \sigma^{2}}$,

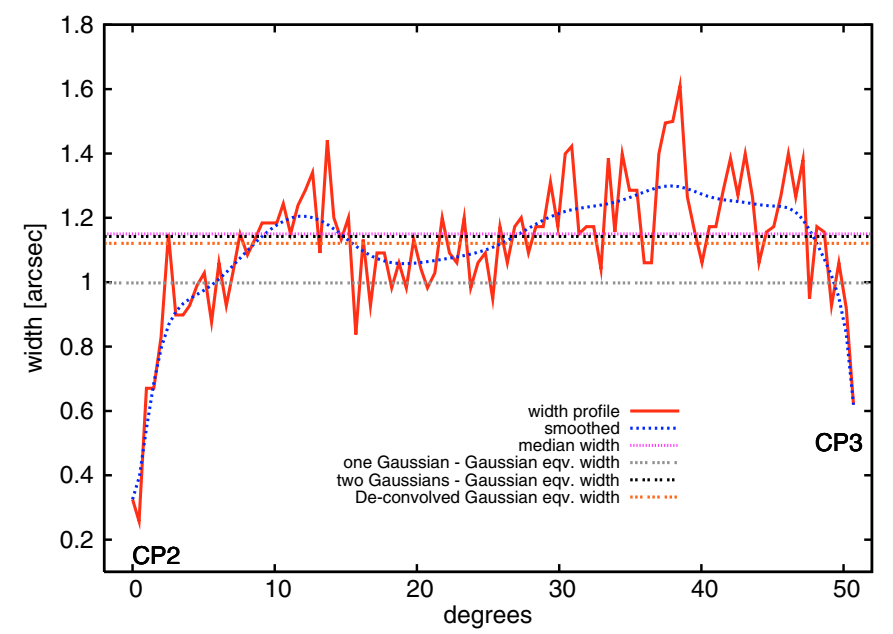

Fig. 13. Transversal width profile of arc A1 (red solid line). The blue dotted line shows an interpolation of the profile with a Bezier curve. The horizontal lines correspond to various determinations of the arc width.

where $\bar{x}$ is the radial distance from the arc center of curvature. We define "Gaussian equivalent width" $W_{\mathrm{G}}$ given by

$W_{\mathrm{G}} \equiv 2 \sqrt{2 \ln \frac{Q}{D}} \sigma$.

In this equation, $D$ represents the detection limit used to select the arc points.

We assume that the total PSF (instrumental PSF+seeing) can be described by another Gaussian function, whose FWHM is equivalent to the PSF size:

$$
G_{\mathrm{PSF}}=\frac{1}{\sqrt{2 \pi} \sigma_{\mathrm{PSF}}} \exp -\frac{x^{2}}{2 \sigma_{\mathrm{PSF}}^{2}},
$$

$F W H M=2 \sqrt{2 \ln 2} \sigma_{\mathrm{PSF}} \simeq 2.3548 \sigma_{\mathrm{PSF}}$.

We further assume that the intrinsic radial profile of the arc is also Gaussian:

$G_{0}(x)=Q_{0} \exp -\frac{(x-\bar{x})^{2}}{2 \sigma_{0}^{2}}$ 


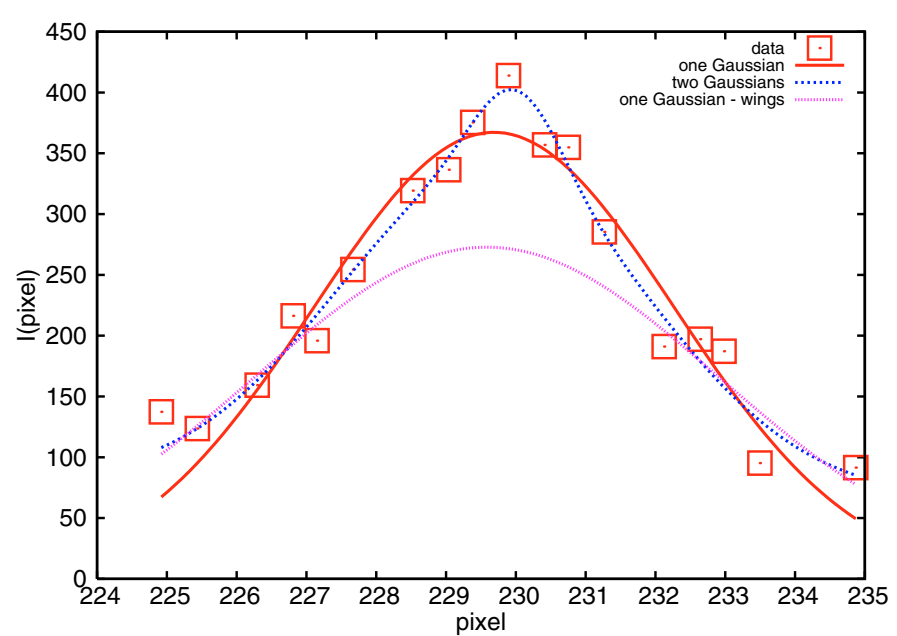

Fig. 14. Median radial profile of arc A1 (square data points). Several attempts of fitting it are shown: single Gaussian (solid line), two Gaussians (long-dotted line) and single Gaussian fitting only the data points below half of the maximum of the profile (short-dotted line).

Then, the observed profile is a simple convolution of two Gaussians, and the parameters of the intrinsic profile can be derived from the observed profile via the equations

$$
\begin{aligned}
& \sigma_{0}=\sqrt{\sigma^{2}-\left(\frac{F W H M}{2.335}\right)^{2}}, \\
& Q_{0}=Q \frac{\sigma}{\sigma_{0}} .
\end{aligned}
$$

Using Eq. (35), the de-convolved Gaussian-equivalent width is

$$
W_{\mathrm{G}, 0}=2 \sqrt{2 \ln \frac{Q_{0}}{D}} \sigma_{0} .
$$

Unfortunately, the radial profile of arcs is not well-fitted by single Gaussians. In Fig. 14, we show the median radial profile of the arc A1. The data points are given by squares. The solid line shows the best single Gaussian fit to the data. Clearly, a single Gaussian provides a bad fit, both in the center and in the wings of the profile. In particular, the fit is lower in the wings than the data. This implies that the Gaussian-equivalent width underestimates the width of the arc. To be consistent with our definition of the length, this is the maximal radial extension of the arc above a given brightness threshold. In this case, the threshold corresponds to $\sim 100$ ADUs. At that level, the Gaussian-equivalent width is $\sim 2$ pixels (i.e. $\sim 0.23^{\prime \prime}$ ) less than the true width.

The fit improves if a combination of Gaussians are used:

$\tilde{G}(x)=\sum_{i=1}^{M} Q_{i} \exp -\frac{\left(x-\bar{x}_{i}\right)^{2}}{2 \sigma_{i}^{2}}$.

The coarsely-dotted line in Fig. 14 shows the best fit obtained by combining $M=2$ Gaussians. The reduced $\chi^{2}$ is smaller by a factor of $\sim 2$ in this case. The resulting fit consists of a combination of a broad Gaussian describing the wings of the profile and of a narrow one describing its central part and contributing very little to the wings. This suggests that, in order to measure the width of the arc, we could use a single Gaussian to fit the external parts of the profile, without affecting the measurements significantly. For example, the Gaussian that fits only the data points below the half of the maximum of the profile is shown by the finely-dotted line. Comparing to the previously described
Table 1. Summary of the main properties of the arcs shown in Fig. 11.

\begin{tabular}{cccccccc}
\hline \hline arc & $L$ & $W_{\max }$ & $W_{\text {med }}$ & $W_{\mathrm{G}}$ & $W_{\mathrm{G}, 0}$ & $R_{\mathrm{c}}$ & $W_{0}$ \\
\hline $\mathrm{A} 1$ & 23.2 & 1.61 & 1.15 & 1.14 & 1.12 & 26.2 & 1.13 \\
$\mathrm{~B} 1$ & 14.3 & 0.98 & 0.81 & 0.81 & 0.77 & 31.5 & 0.7 \\
$\mathrm{C} 1$ & 4.55 & 0.88 & 0.73 & 0.76 & 0.7 & 70.6 & 0.69 \\
$\mathrm{D} 1$ & 3.91 & 0.83 & 0.62 & 0.61 & 0.59 & 1000 & 0.65 \\
$\mathrm{E} 1$ & 8.04 & 1.17 & 0.93 & 0.94 & 0.86 & 18.5 & 0.73 \\
$\mathrm{~F} 1$ & 4.2 & 0.87 & 0.48 & 0.45 & 0.44 & 1000 & 0.47 \\
\hline
\end{tabular}

two-Gaussian fit, the differences in the wings of the profile are minimal.

Even using multiple Gaussians, the de-convolution of the profile can be done as described above. Indeed, each Gaussian component can be de-convolved individually and summed up to provide a de-convolved profile. The arc width can then be derived straightforwardly.

The same fitting procedure can also be applied to the tangential profile of the arc, in order to correct for the PSF effects on the arc length. However, as explained above, for arcs with large length-to-width ratios, the correction is much less important than for the arc width, since the tangential profile declines much more gently than the transversal profile. For example, the best-fit Gaussians describing the decline of the tangential brightness profile on both sides of arc A1 have $\sigma \gtrsim 15$ pixels. The Gaussian best fitting the wings of the radial profile shown in Fig. 14 has $\sigma=3.4$ pixels. Using Eqs. (35) and (41), the PSF correction amounts to $\sim 2 \%$ for the width and $\lesssim 0.3 \%$ for the length in that example.

The width measurements made on arc A1 are displayed in Fig. 13 using horizontal lines. Results are shown both for the PSF convolved and de-convolved widths. The Gaussianequivalent width obtained by fitting the arc radial profile with a single Gaussian is $0.99^{\prime \prime}$. This is $\sim 15 \%$ smaller than the Gaussian-equivalent width measured by using two Gaussians for fitting the profile $\left(1.14^{\prime \prime}\right)$. The latter is very similar to the true median width $\left(1.15^{\prime \prime}\right)$. Finally, the de-convolved width, obtained from the two-Gaussian fit, is $1.12^{\prime \prime}$.

The analysis made on arc A1 were repeated over the other arc-like images in Fig. 11. We summarize the properties of all these arcs in Table 1. In Col. 2 we list the arc lengths (without PSF corrections), followed by several definitions of arc widths, listed in Cols. 3-6. We report both the maximal and the median widths and the Gaussian-equivalent widths before and after PSFcorrection. In Col. 7, we list the arc curvature radii.

The comparison between the median and the maximal widths shows that large fluctuations of the arc thickness are possible along the azimuthal profiles. Differences of about $30 \%$ are typical, but the maximal width can be even $80 \%$ larger than the median width, as shown for the radial arc F1 (see Table). This image is located in a noisy region, near some cluster galaxies, so the edges of the image are very irregular. This confirms that defining the arc width - and other arc properties that depend on this definition, like the length-to-width ratio - is a critical issue.

The agreement between the Gaussian-equivalent and the median widths is generally good. The PSF-corrections are typically a few percent, as expected given the narrow PSF of DUNE. Some arcs are particularly straight. Two examples are the arcs D1 and F1 that have very large curvature radii. In such cases, we arbitrarily assign a curvature radius of $1000^{\prime \prime}$.

By activating and/or deactivating different features of the simulator, the techniques used to analyze the images can be tested to quantify how reliable are the measurements. In the 

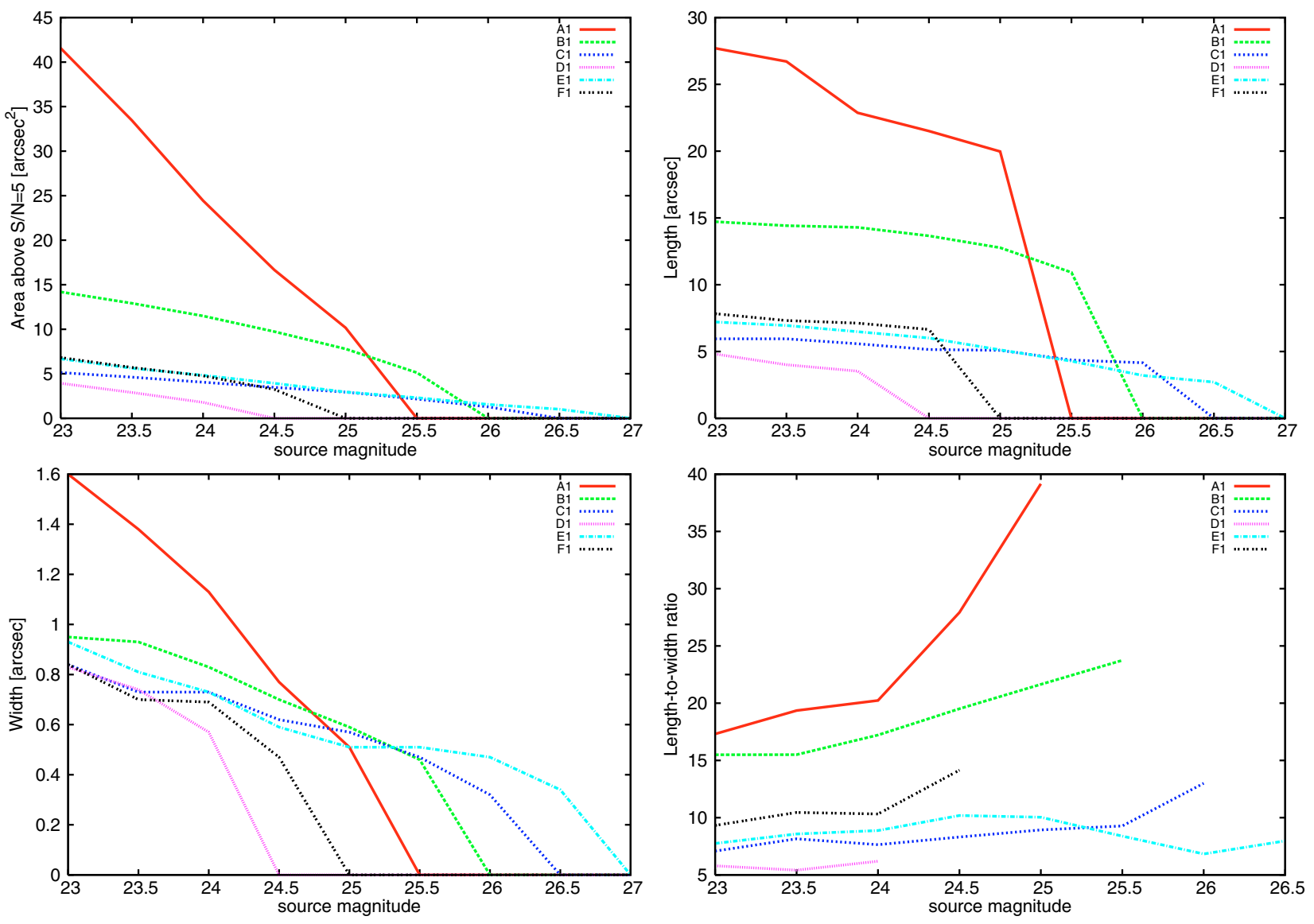

Fig. 15. Arc properties as a function of the source intrinsic magnitude in the riz band. Shown are the area above the minimal signal-to-noise ratio $S / N=5$ (upper-left panel), the length (upper-right panel), the width (bottom-left), and the length-to-width ratio (bottom-right panel). The different line styles correspond to the arcs A1-F1.

last column of Table 1 we show the median widths measured in a simulation where the PSF FWHM is set to zero. The remaining simulation parameters (instrument, exposure time, etc.) are not changed. Although the PSF correction is small, we notice that these newly determined widths are in a slightly better agreement with the PSF-corrected widths in Col. 6 (rms deviation of $\left.\sim 0.16^{\prime \prime}\right)$ than with the previous median or Gaussianequivalent widths in Cols. 4 and 5 ( $\mathrm{rms} \sim 0.23^{\prime \prime}$ and $\sim 0.25^{\prime \prime}$, respectively). This suggests that the de-convolution technique described above has been correctly applied and allows to determine the true widths with an uncertainty of about one pixel.

\subsection{Detectability of arcs}

An important question that can be answered using our simulator is how the properties of the arcs depend on source modeling. First of all, we wish to determine which intrinsic fluxes of the sources make the arcs detectable in DUNE images, assuming an exposure time of $1000 \mathrm{~s}$. For this purpose, we ran several simulations, using the same morphological models of the sources, i.e. without changing their shapelet coefficients, their orientation, and their spectral type, but changing only the input fluxes such that the apparent magnitudes in the riz band vary between $m_{r i z}=23$ and $m_{r i z}=28$. Then, we quantified if the arcs are detectable by counting the number of pixels belonging to the arcs that emerge above a minimal $S / N$ ratio. As before, we fixed this limit to $S / N_{\min }=5$.
The results of this test are shown in Fig. 15 for the arcs $A 1$ to $F 1$ using different line styles. In the top-left panel, we show the arc areas above $S / N_{\min }$ as a function of the intrinsic apparent magnitude of the sources (in the absence of lensing magnification). The limiting source magnitude for which arcs are detectable differs significantly from arc to arc, ranging between 24.5 and 27. The most prominent arcs, i.e. arcs A1 and B1, which are the most magnified sources, can only be detected for source intrinsic magnitudes below 25.5 and 26, respectively, while some shorter arcs, like arcs $\mathrm{C} 1$ and E1, can be detected even if the sources are fainter $\left(m_{r i z} \lesssim 27\right)$. We recall that lensing does not change the surface brightness of the sources, but only the solid angle under which they are observed. Thus, the differences between the detectability limits must reflect the different sizes, shapes and brightness profiles of the sources.

The observed properties of the arcs, in particular the length and the width, obviously depend on the luminosity of the sources. In the top-right and in the bottom-left panels of Fig. 15 we show how the lengths and the widths scale as a function of the intrinsic magnitude. As expected, as the sources become fainter, the arcs shrink in both the tangential and in the radial directions. Such decrements, however, occur at different rates. While the lengths decrease slowly, the change in the arc widths is more rapid. This is clearly due to the presence of several bright knots along the azimuthal profile of the arcs (see e.g. Fig. 13). These originate from multiple images of the same source that merge from opposite sides of the critical lines. Thus, as long as the arcs emerge from the background, their length cannot become shorter than the maximal separation between the knots. Only for very 

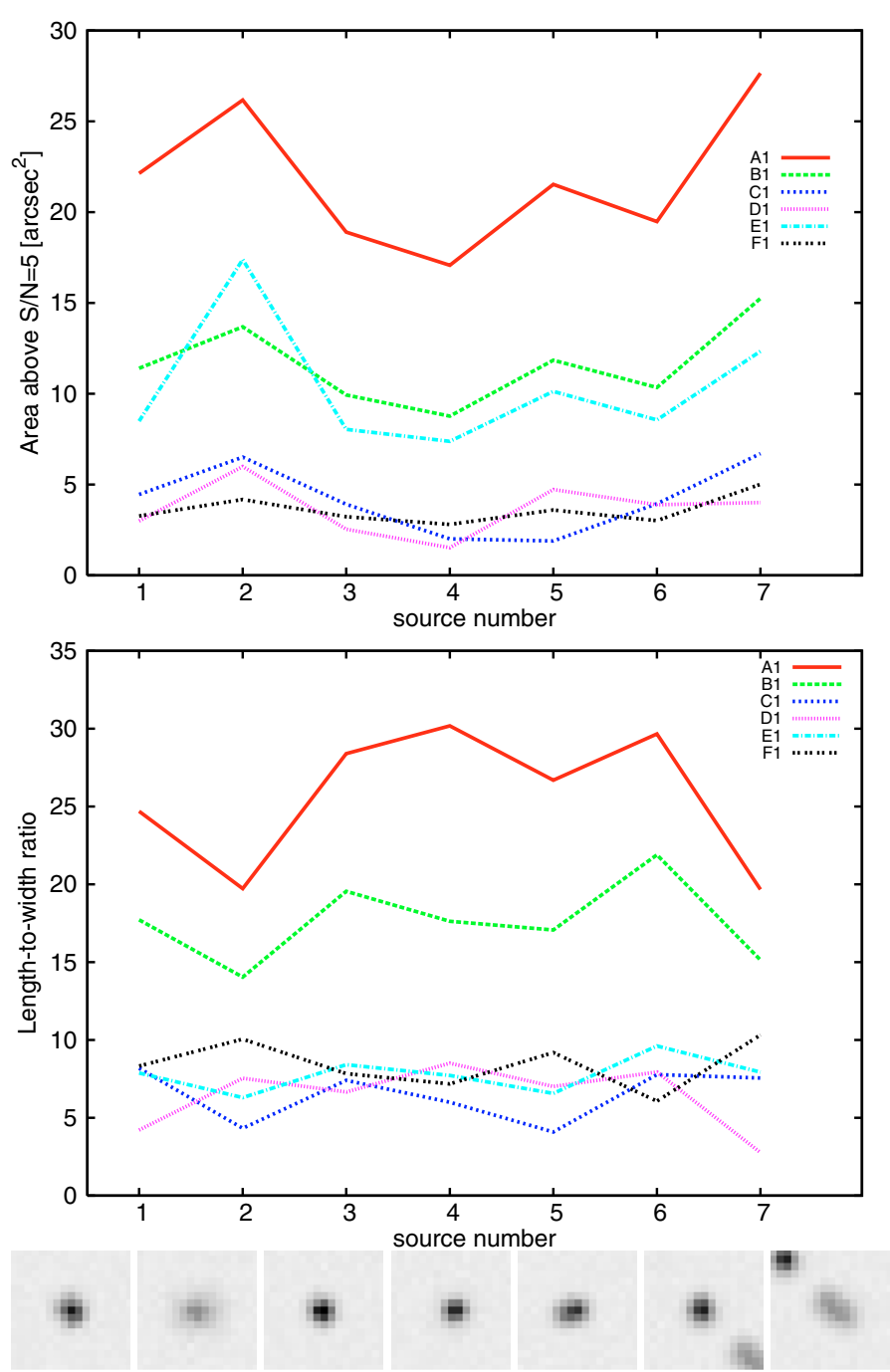

Fig. 16. Top panel: areas of the arcs A1-F1 above $S / N=5$, assuming different sets of shapelet coefficients for modeling the sources. The source models (shown in the figures below the graphs) are numbered from 1 to 7. All sources have intrinsic magnitude $m_{\text {riz }}=24$. Bottom panel: length-to-width ratio as a function of the source model.

faint magnitudes do the arcs break into smaller pieces and the lengths decrease rapidly. This behavior implies that the lengthto-width ratio is not a constant function of the source magnitude. As shown in the bottom-right panel, especially for the longest arcs, the length-to-width ratio is generally inversely proportional to the source luminosity, once the source morphology has been fixed. For example, between $m_{r i z}=23$ and $m_{r i z}=25$, the lengthto-width ratio of the arc A1 becomes greater by more than a factor of two. For arc B1, it grows by $\sim 65 \%$ between $m_{\text {riz }}=23$ and $m_{r i z}=25.5$. Also the length-to-width ratios of the arc $\mathrm{C} 1$ and of the radial arc F1 grow significantly.

To better explain the dependence of the arc appearance on the intrinsic properties of the sources, we ran new simulations, keeping the sources at the same positions but exchanging among them the sets of shapelet coefficients that determine their morphology and surface brightness distributions. In other words, we re-simulated each arc using seven different source shapes. The intrinsic magnitude of the sources was fixed to $m_{\text {riz }}=24$. This value was chosen such that all arcs A1-F1 were detectable. In the upper graph of Fig. 16 we show the area of each arc above $S / N=5$ as a function of the source model. Each model was identified by a number running from 1 to 7 . The corresponding un-lensed images of the sources are shown at the center of the frames below the graphs. Note that these are cut-outs from the left panel of Fig. 11. As anticipated earlier, by varying the morphology of the sources, different amounts of pixels belonging to the arcs surpass the detectability thresholds. In particular, the spatial extent of the arc is determined by the size of the sources, i.e. by their surface brightness. We notice that the curves corresponding to different arcs have many features in common. Indeed, they have several local minima and maxima that arise for the same source models. For example, all arcs have the largest sizes if the source model number 2 or number 7 are used, while their size is the smallest for the source model number 4. Looking at the corresponding source representations, we see that the former are low surface-brightness and spatially-extended galaxies, while the latter corresponds to the most compact among the sources.

We now consider the correlation between the source model and the length-to-width ratio of their images. This is shown in the bottom graph of Fig. 16. Compared to the previous plot, we notice the opposite correlation for most of the images; for a fixed total luminosity of the sources, the most extended galaxies (the sources 2 and 7 in Fig. 16) correspond to the lowest arc lengthto-width ratios.

We can interpret these results as follows. Large, low surface brightness images average magnification over a larger area. Thus, they are less distorted. Indeed, the relative changes in the lengths and of the widths of the images are caused by the convolution of the tangential and of the radial magnifications with source surface brightness distribution. The distortion effects are diluted if the source is broader. For this reason, arcs originating from compact, faint, or low surface brightness sources that are barely detectable are preferentially characterized by high lengthto-width ratios. These results show that by comparing theory to observations, we need to know the source population where the arcs are drawn. As surface brightness is unchanged, we suggest basing the detectability criterion of arcs upon it.

\section{Conclusions}

We presented a new code for mimicking the optical observations of distant galaxies. It includes all relevant sources of noise affecting real observations, such as sky background, photon noise, and the effects of the atmosphere and of the instrumental PSF. The code also incorporates ray-tracing routines that allow us to include lensing effects by matter distributed between the observer and the sources.

In this work we have explained the thorough method that we developed to model the source galaxies realistically. The source morphologies were modeled via the shapelet decomposition of real galaxy images observed with HST. Four spectral templates were used to simulate the spectral energy distributions of the galaxies. Their redshift and luminosity distributions as a function of their spectral classification were derived from observed galaxy luminosity functions in the VVDS. Since it is particularly suitable for simulating lensing by galaxy clusters, the code also simulates the emission from the cluster galaxy population, whose properties are obtained from semi-analytic models of galaxy formation and evolution.

Many features of the simulator were discussed in detail. In particular, we have shown that the code can be easily used to mimic observations with a variety of existing instruments, both from the space (HST) and from the ground (LBT). To illustrate 
the code and to introduce some potential applications, we simulated observations of a galaxy cluster obtained from $N$-body simulations. These virtual observations were made using a telescope with the characteristics of the proposed space telescope DUNE.

The simulated images were analyzed with standard observational techniques. After subtracting the light of the foreground galaxies, multiple images of several distant sources that were strongly lensed by the foreground cluster can be identified. Each detected arc-like image was classified, measuring its length, width, and curvature radius.

We discussed how the properties of arcs can be measured consistently in simulations and observations, in order to facilitate comparison with theoretical predictions. In particular, we focused on the determination of the arc widths and on how they can be corrected for the PSF broadening. For this purpose, we fit the radial profile of the arcs with multiple Gaussians. By comparing simulations with and without PSF convolution, we verified that the de-convolution technique allows one to determine the true width of the arcs with a typical error of one pixel.

By varying the characteristics of the sources, we studied the detectability limits of arcs in DUNE observations. With an exposure time of $1500 \mathrm{~s}$, we expect that DUNE will be able to observe arcs arising from sources whose magnitudes are fainter than $\sim 27$ in the riz band. The shape of the arcs are found to be very sensitive to the properties of the sources. In particular, we found that arcs tend to acquire larger length-to-width ratios as their sources become fainter or more compact.

These results are particularly intriguing for arc statistics. Indeed, they show that observational effects may have a large impact on the abundance of arcs with high length-to width ratios, so they deserve careful investigation. We will dedicate a forthcoming paper to this subject (Meneghetti et al., in prep.). The source morphologies and, in particular, sub-structures of star-forming regions are clearly more relevant for arc statistics than is usually assumed. This is as a result of the small angular size of the bright star-forming regions, which are more easily distorted. Moreover, the number of very elongated images is expected to grow rapidly as a function of the depth of the observations. Thus, space missions like DUNE, capable of making deep and wide surveys thanks to their high sensitivity and wide field of view, are likely to discover a significant number of new gravitational arcs. The use of efficient software for automatic arc identification (see e.g. Lenzen et al. 2005; Seidel \& Bartelmann 2007; Alard 2006; Horesh et al. 2005; Cabanac et al. 2007) will facilitate detection of strong lensing events in these large data sets.

Acknowledgements. The $N$-body simulations were performed at the "Centro Interuniversitario del Nord-Est per il Calcolo Elettronico" (CINECA, Bologna), with CPU time assigned under an INAF-CINECA grant. This work was supported by the Vigoni program of the German Academic Exchange Service (DAAD) and Conference of Italian University Rectors (CRUI). We acknoweldge financial contribution from contracts ASI-INAF I/023/05/0, ASIINAF I/088/06/0, and INFN PD51. C.H. acknowledges the support of a European Commission Program 6th framework Marie Curie Outgoing International Fellowship, under contract M01F-CT-2006-21891. M.M. thanks M. Bolzonella, G. Rodighiero, E. Zucca, S. Bardelli, E. Vanzella, \& G. Zamorani for helpful discussions. We thank A. Refregier, J. Rhodes and the whole DUNE collaboration for allowing us to test our code during the preparation of the DUNE proposal. We thank M. Barden \& S. Koposov, the GOODS and COMBO-17 teams for providing the GOODS galaxy images used for the presented work. We are grateful to C. Peng for providing the software GALFIT and to C. Wolf \& K. Meisenheimer for giving us the SEDs of the GOODS galaxies. We warmly thank V. Springel for providing us with post-processing software. MM thanks Paolo for his cooperation during the preparation of the manuscript.

\section{References}

Alard, C. 2006, ArXiv Astrophysics e-prints

Arnouts, S., Cristiani, S., Moscardini, L., et al. 1999, MNRAS, 310, 540

Aubert, D., Amara, A., \& Metcalf, R. B. 2007, MNRAS, 376, 113

Bartelmann, M., \& Meneghetti, M. 2004, A\&A, 418, 413

Bartelmann, M., \& Schneider, P. 2001, Phys. Rep., 340, 291

Bartelmann, M., Huss, A., Colberg, J., Jenkins, A., \& Pearce, F. 1998, A\&A, 330, 1

Bartelmann, M., Meneghetti, M., Perrotta, F., Baccigalupi, C., \& Moscardini, L. 2003, A\&A, 409, 449

Beckwith, S. V. W., Stiavelli, M., Koekemoer, A. M., et al. 2006, AJ, 132, 1729 Bertin, E., \& Arnouts, S. 1996, A\&AS, 117, 393

Bradac, M., Schneider, P., Lombardi, M., \& Erben, T. 2004, ArXiv Astrophysics e-prints

Cabanac, R. A., Alard, C., Dantel-Fort, M., et al. 2007, A\&A, 461, 813

Cacciato, M., Bartelmann, M., Meneghetti, M., \& Moscardini, L. 2006, A\&A, 458,349

Clowe, D., Gonzalez, A., \& Markevitch, M. 2004, ApJ, 604, 596

Comerford, J. M., Meneghetti, M., Bartelmann, M., \& Schirmer, M. 2006, ApJ, 642, 39

Dalal, N., Hennawi, J. F., \& Bode, P. 2005, ApJ, 622, 99

De Lucia, G., \& Blaizot, J. 2007, MNRAS, 375, 2

Diego, J. M., Tegmark, M., Protopapas, P., \& Sandvik, H. B. 2007, MNRAS, 375,958

Dolag, K., Vazza, F., Brunetti, G., \& Tormen, G. 2005, MNRAS, 364, 753

Ellis, R., Santos, M. R., Kneib, J.-P., \& Kuijken, K. 2001, ApJ, 560, L119

Fedeli, C., Meneghetti, M., Bartelmann, M., Dolag, K., \& Moscardini, L. 2006, A\&A, 447, 419

Fort, B., Prieur, J. L., Mathez, G., Mellier, Y., \& Soucail, G. 1988, A\&A, 200, L17

Giavalisco, M., Sahu, K., \& Bohlin, R. 2002, in HST Instrument Science Report WFC3 2002-12, 1

Giavalisco, M., Ferguson, H. C., Koekemoer, A. M., et al. 2004, ApJ, 600, L93

Gladders, M., Hoekstra, H., Yee, H., Hall, P., \& Barrientos, L. 2003, ApJ, 593, 48

Goldberg, D. M., \& Bacon, D. J. 2005, ApJ, 619, 741

Golse, G., Kneib, J.-P., \& Soucail, G. 2002, A\&A, 387, 788

Grazian, A., Fontana, A., De Santis, C., et al. 2004, PASP, 116, 750

Heymans, C., Van Waerbeke, L., Bacon, D., et al. 2006, MNRAS, 368, 1323

Hilbert, S., White, S. D. M., Hartlap, J., \& Schneider, P. 2007, MNRAS, 927

Hockney, R., \& Eastwood, J. 1988, Computer simulation using particles (Bristol: Hilger)

Horesh, A., Ofek, E. O., Maoz, D., et al. 2005, ApJ, 633, 768

Ilbert, O., Tresse, L., Zucca, E., et al. 2005, A\&A, 439, 863

Kelly, B. C., \& McKay, T. A. 2004, AJ, 127, 625

Kneib, J.-P., Ellis, R. S., Santos, M. R., \& Richard, J. 2004, ApJ, 607, 697

Knuth, D. 1986, Metafont: the Program (Addison-Wesley)

Le Fèvre, O., Vettolani, G., Garilli, B., et al. 2005, A\&A, 439, 845

Lenzen, F., Scherzer, O., \& Schindler, S. 2005, A\&A, 443, 1087

Li, G.-L., Mao, S., Jing, Y. P., et al. 2005, ApJ, 635, 795

Li, G., Zhang, P., \& Chen, X. 2007, ArXiv Astrophysics e-prints

Luppino, G., Gioia, I., Hammer, F., Le Fèvre, O., \& Annis, J. 1999, A\&AS, 136, 117

Lynds, R., \& Petrosian, V. 1989, ApJ, 336, 1

Massey, R., Refregier, A., Conselice, C. J., David, J., \& Bacon, J. 2004, MNRAS, 348, 214

Massey, R., Heymans, C., Bergé, J., et al. 2007a, MNRAS, 376, 13

Massey, R., Rowe, B., Refregier, A., Bacon, D. J., \& Bergé, J. 2007b, MNRAS, 649

Melchior, P., Meneghetti, M., \& Bartelmann, M. 2007, A\&A, 463, 1215

Meneghetti, M., Bolzonella, M., Bartelmann, M., Moscardini, L., \& Tormen, G. 2000, MNRAS, 314, 338

Meneghetti, M., Yoshida, N., Bartelmann, M., et al. 2001, MNRAS, 325, 435

Meneghetti, M., Bartelmann, M., \& Moscardini, L. 2003, MNRAS, 346, 67

Meneghetti, M., Bartelmann, M., Jenkins, A., \& Frenk, C. 2005a, ArXiv Astrophysics e-prints

Meneghetti, M., Jain, B., Bartelmann, M., \& Dolag, K. 2005b, MNRAS, 362, 1301

Meneghetti, M., Bartelmann, M., Dolag, K., et al. 2005c, A\&A, 442, 413

Meneghetti, M., Argazzi, R., Pace, F., et al. 2007, A\&A, 461, 25

Miralda-Escude, J. 1993, ApJ, 403, 497

Oguri, M., Lee, J., \& Suto, Y. 2003, ApJ, 599, 7

Pace, F., Maturi, M., Meneghetti, M., et al. 2007, A\&A, 471, 731 
Paltani, S., Le Fèvre, O., Ilbert, O., et al. 2007, A\&A, 463, 873

Paulin-Henriksson, S., Antonuccio-Delogu, V., Haines, C. P., et al. 2007, A\&A, 467, 427

Peng, C. Y., Ho, L. C., Impey, C. D., \& Rix, H.-W. 2002, AJ, 124, 266

Puchwein, E., Bartelmann, M., Dolag, K., \& Meneghetti, M. 2005, A\&A, 442, 405

Refregier, A. 2003, MNRAS, 338, 35

Rhodes, J. D., Massey, R., Albert, J., et al. 2007, ArXiv Astrophysics e-prints

Sand, D. J., Treu, T., Ellis, R. S., \& Smith, G. P. 2005, ApJ, 627, 32

Sand, D. J., Treu, T., Smith, G. P., \& Ellis, R. S. 2004, ApJ, 604, 88

Schaerer, D. \& Pello, R. 2003, in BAAS, 35, BAAS, 1308
Schechter, P. 1976, ApJ, 203, 297

Seidel, G., \& Bartelmann, M. 2007, A\&A, 472, 341

Simien, F., \& de Vaucouleurs, G. 1986, ApJ, 302, 564

Springel, V. 2005, MNRAS, 364, 1105

Springel, V., White, S. D. M., Tormen, G., \& Kauffmann, G. 2001, MNRAS,

$$
\text { 328, } 726
$$

Starck, J.-L., Pires, S., \& Réfrégier, A. 2006, A\&A, 451, 1139

Torri, E., Meneghetti, M., Bartelmann, M., et al. 2004, MNRAS, 349, 476

Wambsganss, J., Bode, P., \& Ostriker, J. 2004, ApJ, 606, L93

Zaritsky, D., \& Gonzalez, A. 2003, ApJ, 584, 691

Zucca, E., Ilbert, O., Bardelli, S., et al. 2006, A\&A, 455, 879 